\title{
Associations of Serum Lipids and Lipoproteins with the Estimated Glomerular Filtration Rate in the Adult Chinese Population: A Cross-sectional Study
}

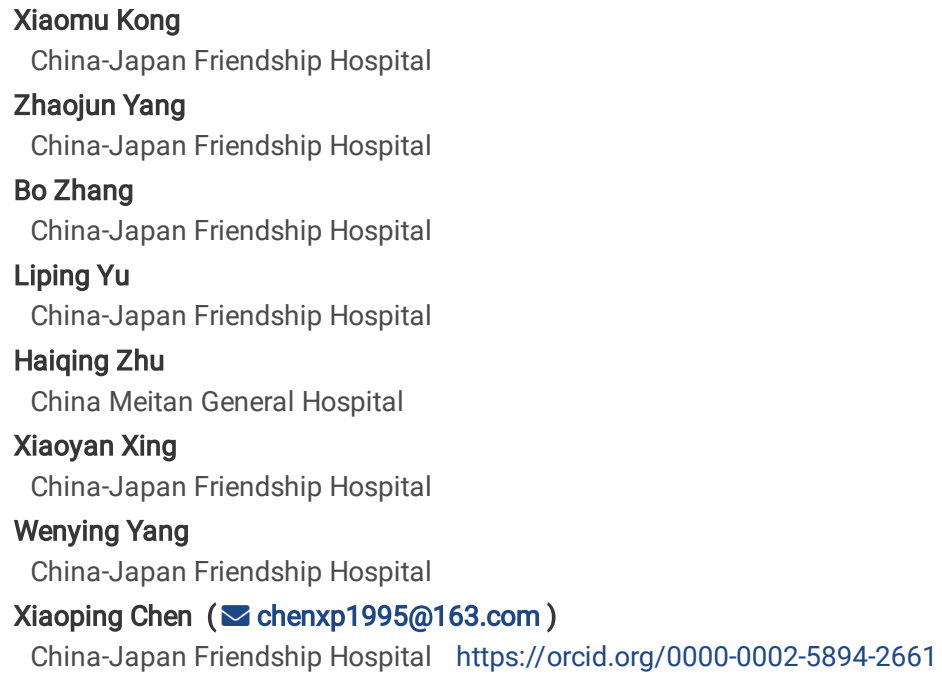

Keywords: Total cholesterol, Triglycerides, High-density lipoprotein-cholesterol, Low-density lipoprotein-cholesterol, Dyslipidemias, Estimated glomerular filtration rate

Posted Date: April 27th, 2020

DOI: https://doi.org/10.21203/rs.3.rs-24670/v1

License: (9) (7) This work is licensed under a Creative Commons Attribution 4.0 International License. Read Full License 


\section{Abstract}

Background: Dyslipidemia has a critical impact on renal dysfunction, but the exact types of lipids and lipoproteins that influence the estimated glomerular filtration rate (eGFR) are under investigation. Observations from an adult Chinese population $\geq 20$ years old are lacking. The present study is to investigate the associations of total cholesterol (TC), triglycerides (TG), high-density lipoprotein-cholesterol (HDL-C), and low-density lipoprotein-cholesterol (LDL-C) with eGFR, and the risks for a mildly and a moderately/severely reduced eGFR.

Methods: A total of 16,206 participants from a national representative adult population (20 years of age or older) in China were included. All calculations were weighted based on the Chinese population data and the sampling scheme.

Results: Upon stratifying the population based on lipids or lipoprotein categories, we observed a significantly lower mean eGFR as well as a higher estimated prevalence of impaired eGFR among Chinese adults with TG levels in the ranges of $1.7 \mathrm{mmol} / \mathrm{L} \leq \mathrm{TG}<2.3 \mathrm{mmol} / \mathrm{L}$ [mean eGFR: $110.99 \mathrm{~mL} / \mathrm{min}$ per $1.73 \mathrm{~m} 2$, $P=0.0000$; prevalence of mildly eGFR impairment: $30.92 \%, P=0.0000$; prevalence of moderately/severely eGFR impairment: $2.01 \%, P=0.1842$ ] and $\geq 2.3$ $\mathrm{mmol} / \mathrm{L}[111.62 \mathrm{~mL} / \mathrm{min}$ per $1.73 \mathrm{~m} 2, \mathrm{P}=0.0000 ; 27.92 \%, \mathrm{P}=0.0000 ; 2.81 \%, \mathrm{P}=0.0174]$, compared to those among individuals with normal TG [122.03 $\mathrm{mL} / \mathrm{min}$ per $1.73 \mathrm{~m} 2 ; 19.96 \% ; 1.20 \%$. Also, a higher prevalence of mildly impaired eGFR was observed with $3.4 \mathrm{mmol} / \mathrm{L} \leq \mathrm{LDL}-\mathrm{C}<4.1 \mathrm{mmol} / \mathrm{L}$ ( $27.93 \%$ ) compared with LDL-C $<3.4 \mathrm{mmol} / \mathrm{L}(22.61 \%, \mathrm{P}=0.0092)$. An elevated TG was proven to be an independent risk factor for decreased eGFR [ $\beta(\mathrm{SE})$ -0.0238(0.0039), $P=0.0000$ ], and each per $\mathrm{mmol} / \mathrm{L}$ increase in TG was associated with the 1.19-fold and 1.31-fold increased risks for mildly impaired eGFR and moderately/severely impaired eGFR, respectively $(P=0.0000,0.0001)$. A decreased HDL-C also showed a negative contribution to eGFR $[-0.0883(0.0176)$, $P=0.0000]$. In contrast, increased TC was associated with increased eGFR [0.0314(0.0060), $P=0.0000]$ and a reduction in the risk for mildly impaired eGFR by 0.91 -fold $(P=0.0302)$. Increased LDL-C also contributed to an elevated eGFR [0.0251(0.0070), $P=0.0004]$.

Conclusion: A decreased eGFR could be attributed independently to an elevated TG or reduced HDL-C. It emphasizes the importance of effective control of TG and HDL-C for preserving eGFR.

\section{Introduction}

Chronic kidney disease (CKD) is a growing health burden that already affects $10 \sim 15 \%$ of adults worldwide. Based on a recent nationwide survey in China, the prevalence of CKD has reached $10.8 \%$ and is still increasing(1). Epidemiological evidence revealed that CKD substantially increases the risk for end-stage renal disease (ESRD) and cardiovascular events, and, strikingly, even a mildly decreased estimated glomerular filtration rate (eGFR; $60 \sim 89 \mathrm{~mL} / \mathrm{min}$ per $1.73 \mathrm{~m}^{2}$ ) could be associated with increased risks for renal function decline and cardiovascular morbidity $(2,3)$. Therefore, the exploration of risk factors for impaired eGFR in the general population is of clinical significance for the early prevention and management of renal dysfunction, which could eventually help to relieve the heavy burden of CKD.

Dyslipidemia has a critical impact on renal dysfunction. Increasing evidence indicates that dyslipidemia is not only associated with a lower eGFR and higher risk for CKD, but also contributes to the risk for a mildly reduced eGFR in the general population. In the Chinese population, the REACTION study based on individuals over 40 years old reported that dyslipidemia increases the risk for CKD by 1.43- and 1.28-fold in men and women, respectively, as well as the risk for an eGFR of $60 \sim 74 \mathrm{~mL} / \mathrm{min}$ per $1.73 \mathrm{~m}^{2}$ by 1.16- and 1.06-fold, respectively(2). Clinical trials also have suggested that approaches targeting dyslipidemia (e.g., statins) could be beneficial for the prevention and management of declining renal function $(4,5)$.

The exact types of lipids and lipoproteins that influence the eGFR are still under investigation, separately. Previous studies established negative correlations between the eGFR and hypercholesterolemia, hypertriglyceridemia, and a reduced high-density lipoprotein-cholesterol (HDL-C) level. For example, Zheng et al. found that elevated total cholesterol (TC) and triglycerides (TG), as well as reduced HDL-C, were associated with the eGFR in participants $\geq 35$ years of age from rural China(6). With adjustment for the other conventional risk factors for eGFR, Sun et al. reported that an elevated TG and a reduced HDL-C were independently associated with the increased risk for CKD defined by an eGFR $60 \mathrm{ml} / \mathrm{min}$ per $1.73 \mathrm{~m}^{2}$ and the presence of albuminuria in a group of patients aged $\geq 40$ years from Guangzhou province in south China(7). Hou et al. reported that TC and TG showed negative contributions to eGFR, while increased TG was associated with mildly reduced eGFR $\left(60 \sim 90 \mathrm{~mL} / \mathrm{min}\right.$ per $\left.1.73 \mathrm{~m}^{2}\right)$ in another group of patients aged $\geq 40$ years with normal serum lipids in China(8). Prospective studies showed that an increased TG and a lower HDL-C can serve as independent predictive factors for eGFR decline and CKD(9-11). However, other studies have yielded conflicting results. For instance, Rahman et al. reported an inverse relationship between TC and low-density lipoprotein-cholesterol (LDL-C) and renal disease outcome in CKD patients with low levels of proteinuria(12). Thus, we speculated that differences in study design and the sampling scheme could confound the associations and limit the generalization of these findings. Observations from the nationwide representative general population of Chinese adults are still lacking.

In the present study, by weighting the data from 16,206 participants recruited from a national representative population (20 years of age or older) in the China National Diabetes and Metabolism Disorders Study (DMS)(13) on the basis of the population data and sampling scheme, we estimated the mean eGFR and prevalence rates of mildly and moderately/severely impaired eGFR according to the four available measurements of lipid and lipoprotein levels (TC, TG, HDL-C and LDL-C) divided into categories and examined the associations of these lipids or lipoproteins with eGFR as well as with the risks for mildly and moderately/severely impaired eGFR. Furthermore, the interactions of these lipids and lipoproteins with conventional factors were explored to pursue a personalized risk assessment and prevention strategy for renal function decline.

\section{Materials And Methods Study participants}


From June 2007 to May 2008, the DMS was carried out using a complex multistage, stratified sampling method, and the detailed methods were described elsewhere(13). In brief, representative regions (including 152 urban districts and 112 rural villages) were selected across China by considering the geographical distribution, economic development, and level of urbanization. Residents aged 20 years and older and who had lived locally for 5 years or more were randomly selected from each region. A total of 16,206 participants, including 6,553 men and 9,653 women, with complete data for eGFR were included in the present study.

\section{Ethics statement}

The study protocol was conducted in accordance with the Declaration of Helsinki II and approved by the ethics committee of the China-Japan Friendship Hospital (Beijing, China). Written informed consent was obtained from each participant prior to data collection.

\section{Clinical and laboratory measurements}

A standardized questionnaire was used to collect information during an interview by well-trained staff. Demographical features and anthropometric characteristics were carefully examined as previously described(13). Body mass index (BMI) was calculated as weight/height ${ }^{2}\left(\mathrm{~kg} / \mathrm{m}^{2}\right)$. Resting blood pressure was measured twice consecutively, and the average systolic blood pressure (SBP) and average diastolic blood pressure (DBP) were calculated and used for analyses.

At enrollment, each participant completed a 75-g oral glucose tolerance test (OGTT) after overnight fasting. The concentrations of TC, TG, HDL-C, and LDL-C, as well as creatine, in fasting serum samples were examined using an automatic biochemical analyzer (Olympus, Tokyo, Japan). The urinary albumincreatinine ratio (UACR) was measured using a DCA Vantage ${ }^{T M}$ analyzer (Siemens, Erlangen, Germany) utilizing an immunoassay method for albumin. The eGFR was calculated using the abbreviated Modification of Diet in Renal Disease (MDRD) equation for the Chinese population: eGFR ( $\mathrm{mL} / \mathrm{min}$ per $1.73 \mathrm{~m}{ }^{2}$ ) = $186 \times$ [serum creatine $(\mu \mathrm{mol} / \mathrm{L}) / 88.4]^{-1.154} \times$ age $^{-0.203} \times 1.233 \times[0.742$ for women $](14)$.

\section{Definitions}

Normal renal function was defined by an eGFR $\geq 90 \mathrm{ml} / \mathrm{min}$ per $1.73 \mathrm{~m}^{2}$. Mild eGFR impairment was defined by $60 \leq \mathrm{eGFR}<90 \mathrm{ml} / \mathrm{min}$ per $1.73 \mathrm{~m}{ }^{2}$. Moderate/severe eGFR impairment was defined by eGFR $<60 \mathrm{ml} / \mathrm{min}$ per $1.73 \mathrm{~m}^{2}(15)$.

The blood lipid levels were classified into categories according to the guidelines for the prevention and treatment of dyslipidemia in Chinese adults. TG was divided into three categories (normal: $<1.7 \mathrm{mmol} / \mathrm{L}$, borderline high: $\geq 1.7$ and $<2.3 \mathrm{mmol} / \mathrm{L}$, high: $\geq 2.3 \mathrm{mmol} / \mathrm{L}$ ), TC was divided into three categories (normal: $<5.2 \mathrm{mmol} / \mathrm{L}$, borderline high: $\geq 5.2$ and $<6.2 \mathrm{mmol} / \mathrm{L}$, and high: $\geq 6.2 \mathrm{mmol} / \mathrm{L}$ ), HDL-C was divided into two categories (normal: $\geq 1.0 \mathrm{mmol} / \mathrm{L}$ and low: $<1.0 \mathrm{mmol} / \mathrm{L}$ ), and LDL-C was divided into three categories (normal: $<3.4 \mathrm{mmol} / \mathrm{L}$, borderline high: $\geq 3.4$ and $<4.1 \mathrm{mmol} / \mathrm{L}$, high: $\geq 4.1 \mathrm{mmol} / \mathrm{L}$ ) $(16-18)$.

Underweight was defined by a BMI $<18.5 \mathrm{~kg} / \mathrm{m}^{2}$, normal weight was defined by a BMI $18.5 \sim 24 \mathrm{~kg} / \mathrm{m}^{2}$, and overweight/obese was defined by a BMI $\geq$ $24 \mathrm{~kg} / \mathrm{m}^{2}(16)$.

Diabetes was identified according to the 1999 World Health Organization (WHO) criteria of fasting plasma glucose (FPG) $\geq 7.0 \mathrm{mmol} / \mathrm{L}, 2-\mathrm{h}$ post-prandial plasma glucose (2-h PPG) $\geq 11.1 \mathrm{mmol} / \mathrm{L}$, or a self-reported history of diabetes. Prediabetes was defined by FPG $\geq 6.1 \mathrm{mmol} / \mathrm{L}$ and $<7.0 \mathrm{mmol} / \mathrm{L}$ and/or $2-\mathrm{h}$ $P P G \geq 7.8 \mathrm{mmol} / \mathrm{L}$ and $<11.1 \mathrm{mmol} / \mathrm{L}$, with no previous diagnosis of diabetes.

Hypertension was defined by an SBP $\geq 140 \mathrm{mmHg}$ and/or mean DBP $\geq 90 \mathrm{mmHg}$, or receiving anti-hypertension therapy. Prehypertension was defined by SBP of $120 \sim 140 \mathrm{mmHg}$ or DBP of $80 \sim 90 \mathrm{mmHg}$ with no previous diagnosis of hypertension(19).

\section{Statistical analysis}

All statistical analyses were conducted with the use of SUDAAN software (version 10; Research Triangle Institute, NC, USA). Using SUDAAN, all calculations in this study were weighted to represent the total population of Chinese adults (20 years of age or older) on the basis of Chinese population data from 2006 and the study sampling scheme as previously described(13). Oversampling of female and urban residents, non-response, economic development, and other demographic or geographic differences between the sample and the total population of the survey were taken into account. The reported $P$ values were twotailed, and $P<0.05$ was considered as significant.

The mean values and $95 \%$ confidence intervals (Cls) for the clinical features and estimated prevalence rates of metabolic disorders were determined according to eGFR categories. The mean values of eGFR and the prevalence estimates for mildly impaired eGFR and moderately/severely impaired eGFR according to the TC, TG, HDL-C, and LDL-C categories were calculated. The differences in means and frequencies between categories were tested using the PAIRWISE procedure in SUDAAN software.

Regression analysis (REGRESS procedure) was applied to determine the effects of TC, TG, HDL-C, and LDL-C, as well as their categories, on eGFR. The beta coefficient ( $\beta$ ) and standard error (SE) were calculated. Prior to the regression analyses, eGFR was natural logarithmically transformed. A multinomial logistic analysis (MULTILOG procedure) was used to test the associations of TC, TG, HDL-C, and LDL-C, as well as their categories, with the risks for mildly impaired eGFR and moderately/severely impaired eGFR. The odds ratio (OR) and $95 \% \mathrm{Cl}$ were calculated. For the above calculations, the conventional risk factors for eGFR, including gender, age, BMl, smoking status (no/yes), alcohol intake habit (no/yes), diabetes status (normal glycemic metabolism/prediabetes/diabetes), and hypertension status (normal tension/prehypertension/hypertension), were adjusted as confounders. To examine the interaction effects of these conventional factors on the above associations, the interaction terms were further included in these models, separately. Moreover, the association studies were also performed in subpopulations according to sex, age ( $<40,40 \sim 60$, and $\geq 60$ years), BMI (underweight, normal weight, vs. overweight/obese), smoking 
status (no vs. yes), alcohol intake habit (no vs. yes), diabetes status (normal glycemic metabolism, prediabetes vs. diabetes), and hypertension status (normal tension, prehypertension vs. hypertension), separately.

\section{Results}

\section{Clinical characteristics of study participants according to eGFR categories}

The clinical characteristics of the participants in the different eGFR categories are shown in Table 1. In total, 16,206 participants were included in the present study. Among them, 13,237 had normal renal function [eGFR $\geq 90 \mathrm{ml} / \mathrm{min}$ per $1.73 \mathrm{~m}^{2}$ : mean $(95 \% \mathrm{Cl}) 132.22(127.44,137.00) \mathrm{ml} / \mathrm{min} \mathrm{per} 1.73 \mathrm{~m}{ }^{2}$ ], $2,780 \mathrm{had}$ a mildly impaired eGFR [eGFR between 60 to $90 \mathrm{ml} / \mathrm{min}$ per $1.73 \mathrm{~m}^{2}: 79.42(78.90,79.94) \mathrm{ml} / \mathrm{min}$ per $1.73 \mathrm{~m}^{2}$ ], and 189 had a moderately/severely impaired eGFR [eGFR $<60 \mathrm{ml} / \mathrm{min}$ per $1.73 \mathrm{~m}^{2}: 48.00(44.36,51.65) \mathrm{ml} / \mathrm{min}$ per $1.73 \mathrm{~m}^{2}$ ] (Table 1$)$. The estimated prevalence rates of mildly impaired eGFR and moderately/severely impaired eGFR were $22.63 \%$ and $1.57 \%$, respectively (Table 2 ). 
Table 1

Clinical characteristics of the study participants according to the estimated glomerular filtration rate categories.

\begin{tabular}{|c|c|c|c|c|c|c|}
\hline Traits & $\begin{array}{l}\text { Normal eGFR } \\
(\text { eGFR } \geq 90 \mathrm{ml} / \mathrm{min} \\
\left.\text { per } 1.73 \mathrm{~m}^{2}\right)\end{array}$ & $\begin{array}{l}\text { Mildly impaired eGFR } \\
\text { (eGFR } 60 \sim 90 \mathrm{ml} / \mathrm{min} \\
\text { per } 1.73 \mathrm{~m}^{2} \text { ) }\end{array}$ & $\begin{array}{l}\text { Moderately/severely } \\
\text { impaired eGFR } \\
(\text { eGFR }<60 \mathrm{ml} / \mathrm{min} \text { per } \\
1.73 \mathrm{~m}^{2} \text { ) }\end{array}$ & $\begin{array}{l}P_{\text {eGFR 60 90 }} \\
\text { vs. eGFR } \geq 90\end{array}$ & $\begin{array}{l}P_{\text {eGFR }<60 \text { vs. }} \\
\text { eGFR } \geq 90\end{array}$ & $\begin{array}{l}P_{\text {eGFR }<60 \text { vs. }} \\
\text { eGFR 60 90 }\end{array}$ \\
\hline $\mathbf{N}$ & 13,237 & 2,780 & 189 & & & \\
\hline $\mathrm{N}_{\text {Male }}$ & 5,581 & 921 & 51 & & & \\
\hline Mean age $(95 \% \mathrm{Cl})$, year & $42.66(42.03,43.30)$ & $52.59(51.74,53.43)^{a}$ & $\begin{array}{l}58.62(54.59,62.65) \\
a, b\end{array}$ & 0.0000 & 0.0000 & 0.0039 \\
\hline Mean BMI (95\% Cl), kg/m² & $23.62(23.51,23.74)$ & $23.99(23.80,24.18)^{a}$ & $23.95(23.21,24.69)$ & 0.0005 & 0.2759 & 0.9707 \\
\hline $\begin{array}{l}\text { Prevalence of underweight } \\
(95 \% \mathrm{Cl}), \%\end{array}$ & $4.92(4.31,5.63)$ & $3.18(2.42,4.15)^{a}$ & $3.40(1.20,9.24)$ & 0.0015 & 0.4001 & 0.9024 \\
\hline $\begin{array}{l}\text { Prevalence of } \\
\text { overweight/obese ( } 95 \% \mathrm{Cl}), \%\end{array}$ & $42.27(40.55,44.02)$ & $47.59(44.84,50.35)^{a}$ & $43.31(28.70,59.19)$ & 0.0014 & 0.8979 & 0.5998 \\
\hline $\begin{array}{l}\text { Mean fasting plasma glucose } \\
(95 \% \mathrm{Cl}), \mathrm{mmol} / \mathrm{L}\end{array}$ & $5.10(5.06,5.14)$ & $5.45(5.37,5.52)^{a}$ & $7.79(4.32,11.25)$ & 0.0000 & 0.0724 & 0.1541 \\
\hline $\begin{array}{l}\text { Mean 2-h OGTT glucose (95\% } \\
\mathrm{Cl}), \mathrm{mmol} / \mathrm{L}\end{array}$ & $6.45(6.35,6.54)$ & $7.60(7.38,7.81)^{a}$ & $11.19(6.28,16.10)^{a}$ & 0.0000 & 0.0145 & 0.1105 \\
\hline $\begin{array}{l}\text { Prevalence of prediabetes } \\
(95 \% \mathrm{Cl}), \%\end{array}$ & $13.43(11.96,15.05)$ & $20.66(18.40,23.13)^{a}$ & $20.81(12.08,33.45)$ & 0.0000 & 0.1805 & 0.9795 \\
\hline $\begin{array}{l}\text { Prevalence of diabetes ( } 95 \% \\
\mathrm{Cl}), \%\end{array}$ & $6.96(6.22,7.80)$ & $13.62(11.74,15.76)^{a}$ & $29.14(12.86,53.41)^{a}$ & 0.0000 & 0.0401 & 0.1524 \\
\hline Mean SBP (95\% Cl), mmHg & $\begin{array}{l}119.82(119.05 \\
120.59)\end{array}$ & $\begin{array}{l}125.30(123.62 \\
126.99)^{a}\end{array}$ & $\begin{array}{l}134.01(128.51 \\
139.50)^{a, b}\end{array}$ & 0.0000 & 0.0000 & 0.0017 \\
\hline Mean DBP (95\% Cl), mmHg & $76.53(75.99,77.06)$ & $77.61(76.57,78.65)$ & $\begin{array}{l}82.81(79.68,85.95) \\
a, b\end{array}$ & 0.0978 & 0.0001 & 0.0015 \\
\hline $\begin{array}{l}\text { Prevalence of prehypertension } \\
(95 \% \mathrm{Cl}), \%\end{array}$ & $36.07(34.46,37.72)$ & $36.81(34.11,39.59)$ & $27.05(17.45,39.42)$ & 0.6522 & 0.1148 & 0.0942 \\
\hline $\begin{array}{l}\text { Prevalence of hypertension } \\
(95 \% \mathrm{Cl}), \%\end{array}$ & $19.64(18.09,21.29)$ & $29.83(27.36,32.42)^{a}$ & $\begin{array}{l}55.00(39.12,69.92) \\
a, b\end{array}$ & 0.0000 & 0.0000 & 0.0022 \\
\hline Mean TG (95\% Cl), mmol/L & $1.48(1.45,1.52)$ & $1.73(1.66,1.79)^{a}$ & $1.94(1.69,2.20)^{a, b}$ & 0.0000 & 0.0000 & 0.0044 \\
\hline $\begin{array}{l}\text { Prevalence of borderline high } \\
\text { TG }(95 \% \mathrm{Cl}), \%\end{array}$ & $12.00(10.92,13.17)$ & $18.49(16.28,20.93)^{a}$ & $17.52(10.08,28.69)$ & 0.0000 & 0.2451 & 0.8413 \\
\hline $\begin{array}{l}\text { Prevalence of high TG }(95 \% \\
\mathrm{Cl}), \%\end{array}$ & $14.02(12.92,15.19)$ & $18.89(16.90,21.05)^{a}$ & $27.73(17.25,41.39)^{a}$ & 0.0001 & 0.0287 & 0.1625 \\
\hline Mean TC (95\% Cl), mmol/L & $4.67(4.63,4.70)$ & $4.81(4.76,4.86)^{a}$ & $5.07(4.69,5.44)^{a}$ & 0.0000 & 0.0351 & 0.1971 \\
\hline $\begin{array}{l}\text { Prevalence of borderline high } \\
\text { TC }(95 \% \mathrm{Cl}), \%\end{array}$ & $21.47(19.86,23.18)$ & $23.85(21.55,26.32)$ & $22.49(13.46,35.11)$ & 0.1082 & 0.8559 & 0.8099 \\
\hline $\begin{array}{l}\text { Prevalence of high TC }(95 \% \\
\text { Cl), } \%\end{array}$ & $6.02(5.25,6.89)$ & $7.89(6.53,9.50)^{a}$ & $22.81(7.37,52.34)$ & 0.0297 & 0.1546 & 0.2067 \\
\hline $\begin{array}{l}\text { Mean HDL-C (95\% Cl), } \\
\mathrm{mmol} / \mathrm{L}\end{array}$ & $1.33(1.32,1.35)$ & $1.35(1.33,1.37)$ & $1.35(1.18,1.53)$ & 0.2135 & 0.9663 & 0.9039 \\
\hline $\begin{array}{l}\text { Prevalence of low HDL-C ( } 95 \% \\
\mathrm{Cl}), \%\end{array}$ & $15.20(13.95,16.54)$ & $13.76(12.02,15.71)$ & $18.51(10.52,30.49)$ & 0.2104 & 0.5168 & 0.3564 \\
\hline
\end{tabular}

Abbreviations: $\mathrm{BMI}$, body mass index; $\mathrm{Cl}$, confidence interval; DBP, diastolic blood pressure; eGFR, estimated glomerular filtration rate; HDL-C, high-density lipoprotein-cholesterol; LDL-C, low-density lipoprotein-cholesterol; OGTT, oral glucose tolerance test; SBP, systolic blood pressure; SE, standard error; TC, total cholesterol; TG, triglycerides; UACR, urinary albumin-creatine-ratio.

For lipids and lipoprotein categories, the following definitions were used. Borderline high TG: TG $\geq 1.7$ and $<2.3 \mathrm{mmol} / \mathrm{L}$. High TG: TG $\geq 2.3 \mathrm{mmol} / \mathrm{L}$ ). Borderline high TC: TC $\geq 5.2$ and $<6.2 \mathrm{mmol} / \mathrm{L}$. High TC: TC $\geq 6.2 \mathrm{mmol} / \mathrm{L}$. Low HDL-C: HDL-C $<1.0 \mathrm{mmol} / \mathrm{L}$. Borderline high LDL-C: LDL-C $\geq 3.4$ and $<$ $4.1 \mathrm{mmol} / \mathrm{L}$. High LDL-C: LDL-C $\geq 4.1 \mathrm{mmol} / \mathrm{L}$.

Data are shown as mean $(95 \% \mathrm{Cl})$ or prevalence $(95 \% \mathrm{Cl})$. All mean values and estimated prevalence rates were weighted to represent the total population of Chinese adults ( $\geq 20$ years old) base on the Chinese population data from 2006 . All non-Gaussian distributed quantitative traits were natural logarithmically transformed prior to the comparisons. The differences of means or prevalence rates between the eGFR categories were tested using the PAIRWISE procedure in SUDAAN software. $P<0.05$ is considered statistically significant. ${ }^{a}$, significant different from eGFR $\geq 90 \mathrm{ml} / \mathrm{min}$ per $1.73 \mathrm{~m}{ }^{2} \mathrm{group}$; ${ }^{b}$, eGFR $<60 \mathrm{ml} / \mathrm{min}$ per $1.73 \mathrm{~m}^{2}$ group is significant different from eGFR $60 \sim 90 \mathrm{ml} / \mathrm{min}$ per $1.73 \mathrm{~m}^{2}$ group. 


\begin{tabular}{|c|c|c|c|c|c|c|}
\hline Traits & $\begin{array}{l}\text { Normal eGFR } \\
(e G F R \geq 90 \mathrm{ml} / \mathrm{min} \\
\left.\text { per } 1.73 \mathrm{~m}^{2}\right)\end{array}$ & $\begin{array}{l}\text { Mildly impaired eGFR } \\
(\text { eGFR } 60 \sim 90 \mathrm{ml} / \mathrm{min} \\
\left.\text { per } 1.73 \mathrm{~m}^{2}\right)\end{array}$ & $\begin{array}{l}\text { Moderately/severely } \\
\text { impaired eGFR } \\
(\text { eGFR }<60 \mathrm{ml} / \mathrm{min} \text { per } \\
\left.1.73 \mathrm{~m}^{2}\right)\end{array}$ & $\begin{array}{l}P_{\text {eGFR } 60 \sim 90} \\
\text { vs. eGFR } \geq 90\end{array}$ & $\begin{array}{l}P_{\text {eGFR } 600 \text { vs. }} \\
\text { eGFR } \geq 90\end{array}$ & $\begin{array}{l}P_{\text {eGFR }}<0 \text { vs. } \\
\text { eGFR } 60 \sim 90\end{array}$ \\
\hline Mean LDL-C (95\% Cl), mmol/L & $2.72(2.69,2.75)$ & $2.84(2.80,2.88)^{a}$ & $3.08(2.81,3.35)^{a}$ & 0.0000 & 0.0072 & 0.0915 \\
\hline $\begin{array}{l}\text { Prevalence of borderline high } \\
\text { LDL-C }(95 \% \mathrm{Cl}), \%\end{array}$ & $13.2(11.87,14.66)$ & $17.02(14.91,19.36)^{a}$ & $24.94(9.36,51.66)$ & 0.0044 & 0.2940 & 0.4803 \\
\hline $\begin{array}{l}\text { Prevalence of high LDL-C } \\
(95 \% \mathrm{Cl}), \%\end{array}$ & $5.51(4.49,6.75)$ & $5.78(4.70,7.09)$ & $10.24(5.00,19.83)$ & 0.7439 & 0.1978 & 0.2256 \\
\hline $\begin{array}{l}\text { Mean eGFR }(95 \% \mathrm{Cl}), \mathrm{ml} / \mathrm{min} \\
\text { per } 1.73 \mathrm{~m}^{2}\end{array}$ & $\begin{array}{l}132.22(127.44, \\
137.00)\end{array}$ & $79.42(78.90,79.94)^{a}$ & $\begin{array}{l}48.00(44.36,51.65) \\
a, b\end{array}$ & 0.0000 & 0.0000 & 0.0000 \\
\hline Mean UACR ( $95 \% \mathrm{Cl}), \mathrm{mg} / \mathrm{g}$ & $27.23(25.14,29.31)$ & $32.19(28.34,36.03)$ & $\begin{array}{l}98.35(64.01,132.70) \\
a, b\end{array}$ & 0.1233 & 0.0000 & 0.0000 \\
\hline \multicolumn{7}{|c|}{$\begin{array}{l}\text { Abbreviations: BMI, body mass index; Cl, confidence interval; DBP, diastolic blood pressure; eGFR, estimated glomerular filtration rate; HDL-C, high-density } \\
\text { lipoprotein-cholesterol; LDL-C, low-density lipoprotein-cholesterol; OGTT, oral glucose tolerance test; SBP, systolic blood pressure; SE, standard error; TC, } \\
\text { total cholesterol; TG, triglycerides; UACR, urinary albumin-creatine-ratio. }\end{array}$} \\
\hline \multicolumn{7}{|c|}{$\begin{array}{l}\text { For lipids and lipoprotein categories, the following definitions were used. Borderline high TG: TG } \geq 1.7 \text { and }<2.3 \mathrm{mmol} / \mathrm{L} \text {. High TG: TG } \geq 2.3 \mathrm{mmol} / \mathrm{L}) \text {. } \\
\text { Borderline high TC: TC } \geq 5.2 \text { and }<6.2 \mathrm{mmol} / \mathrm{L} \text {. High TC: TC } \geq 6.2 \mathrm{mmol} / \mathrm{L} \text {. Low HDL-C: HDL-C }<1.0 \mathrm{mmol} / \mathrm{L} \text {. Borderline high LDL-C: LDL-C } \geq 3.4 \text { and }< \\
4.1 \mathrm{mmol} / \mathrm{L} \text {. High LDL-C: LDL-C } \geq 4.1 \mathrm{mmol} / \mathrm{L} \text {. }\end{array}$} \\
\hline \multicolumn{7}{|c|}{$\begin{array}{l}\text { Data are shown as mean }(95 \% \mathrm{Cl}) \text { or prevalence }(95 \% \mathrm{Cl}) \text {. All mean values and estimated prevalence rates were weighted to represent the total population } \\
\text { of Chinese adults }(\geq 20 \text { years old) base on the Chinese population data from } 2006 \text {. All non-Gaussian distributed quantitative traits were natural } \\
\text { logarithmically transformed prior to the comparisons. The differences of means or prevalence rates between the eGFR categories were tested using the } \\
\text { PAIRWISE procedure in SUDAAN software. } P<0.05 \text { is considered statistically significant. }{ }^{a} \text {, significant different from eGFR } \geq 90 \mathrm{ml} / \mathrm{min} \text { per } 1.73 \mathrm{~m}{ }^{2} \mathrm{group} \\
{ }^{b} \text {, eGFR }<60 \mathrm{ml} / \mathrm{min} \text { per } 1.73 \mathrm{~m}^{2} \text { group is significant different from eGFR } 60 \sim 90 \mathrm{ml} / \mathrm{min} \text { per } 1.73 \mathrm{~m}^{2} \text { group. }\end{array}$} \\
\hline
\end{tabular}


Table 2

Age- and sex-standardized means of the estimated glomerular filtration rate and prevalence rates of estimated glomerular filtration rate impairment in Chinese population.

\begin{tabular}{|c|c|c|c|}
\hline Population & $\begin{array}{l}\text { Mean eGFR }(95 \% \mathrm{Cl}), \mathrm{ml} / \mathrm{min} \text { per } \\
1.73 \mathrm{~m}^{2}\end{array}$ & $\begin{array}{l}\text { Mildly impaired eGFR } \\
(\text { (eGFR } 60 \sim 90 \mathrm{ml} / \mathrm{min} \text { per } \\
1.73 \mathrm{~m}^{2} \text { ) } \\
\text { Estimated prevalence }(95 \% \mathrm{Cl}), \%\end{array}$ & $\begin{array}{l}\text { Moderately/severely impaired eGFR } \\
\left.\text { (eGFR }<60 \mathrm{ml} / \mathrm{min} \text { per } 1.73 \mathrm{~m}^{2}\right) \\
\text { Estimated prevalence }(95 \% \mathrm{Cl}), \%\end{array}$ \\
\hline Overall cohort & $118.95(115.27,122.63)$ & $22.63(21.53,23.78)$ & $1.57(1.12,2.19)$ \\
\hline \multicolumn{4}{|l|}{ TC categories } \\
\hline Normal: $<5.2 \mathrm{mmol} / \mathrm{L}$ & $118.80(113.93,123.68)$ & $21.68(20.41,23.01)$ & $1.17(0.87,1.56)$ \\
\hline $\begin{array}{l}\text { Borderline high: } 5.2 \sim \\
6.3 \mathrm{mmol} / \mathrm{L}\end{array}$ & $118.08(114.02,122.14)$ & $24.52(21.94,27.30)$ & $1.55(0.99,2.43)$ \\
\hline High: $\geq 6.3 \mathrm{mmol} / \mathrm{L}$ & $119.96(107.23,132.69)$ & $26.68(22.07,31.86)$ & $5.19(1.48,16.65)$ \\
\hline \multicolumn{4}{|l|}{ TG categories } \\
\hline Normal: $<1.7 \mathrm{mmol} / \mathrm{L}$ & $122.03(117.00,127.07)$ & $19.96(18.72,21.27)$ & $1.20(0.68,2.10)$ \\
\hline $\begin{array}{l}\text { Borderline high: } 1.7 \sim \\
2.3 \mathrm{mmol} / \mathrm{L}\end{array}$ & $110.99(105.44,116.55)^{a}$ & $30.92(27.37,34.70)^{a}$ & $2.01(1.23,3.28)$ \\
\hline High: $\geq 2.3 \mathrm{mmol} / \mathrm{L}$ & $111.62(107.85,115.39)^{a}$ & $27.92(25.08,30.95)^{a}$ & $2.81(1.87,4.21)^{a}$ \\
\hline \multicolumn{4}{|l|}{ HDL-C categories } \\
\hline Normal: $\geq 1.0 \mathrm{mmol} / \mathrm{L}$ & $118.99(115.10,122.88)$ & $22.92(21.72,24.18)$ & $1.48(0.99,2.21)$ \\
\hline Low: $<1.0 \mathrm{mmol} / \mathrm{L}$ & $119.11(108.15,130.06)$ & $20.85(18.18,23.79)$ & $1.92(1.14,3.19)$ \\
\hline \multicolumn{4}{|l|}{ LDL-C categories } \\
\hline Normal: <3.4 mmol/L & $118.70(114.09,123.31)$ & $22.61(21.38,23.90)$ & $1.32(1.01,1.73)$ \\
\hline $\begin{array}{l}\text { Borderline high: } 3.4 \text { } \\
4.1 \mathrm{mmol} / \mathrm{L}\end{array}$ & $113.12(109.19,117.04)$ & $27.93(24.31,31.86)^{a}$ & $2.85(0.92,8.44)$ \\
\hline High: $\geq 4.1 \mathrm{mmol} / \mathrm{L}$ & $122.44(107.34,137.54)$ & $24.00(19.05,29.76)$ & $2.96(1.49,5.78)$ \\
\hline \multicolumn{4}{|c|}{$\begin{array}{l}\text { Abbreviations: Cl, confidence interval; eGFR, estimated glomerular filtration rate; HDL-C, high-density lipoprotein-cholesterol; LDL-C, low-density lipoprotein- } \\
\text { cholesterol; OR, odds ratio; SE, standard error; TC, total cholesterol; TG, triglycerides. }\end{array}$} \\
\hline \multicolumn{4}{|c|}{$\begin{array}{l}\text { Data are shown as mean }(95 \% \mathrm{Cl}) \text { or prevalence }(95 \% \mathrm{Cl}) \text {. All mean values and estimated prevalence rates were weighted to represent the total population } \\
\text { of Chinese adults }(\geq 20 \text { years old) based on Chinese population data from } 2006 \text {. All non-Gaussian distributed quantitative traits were natural } \\
\text { logarithmically transformed prior to the comparisons. The differences of means or prevalence rates between the categories of TC, TG, HDL-C, and LDL-C } \\
\text { were tested using the PAIRWISE procedure in SUDAAN software. } P<0.05 \text { is considered statistically significant. }{ }^{2} \text {, significant different from the normal } \\
\text { category. } P \text { values }<0.05 \text { are shown in bold. }\end{array}$} \\
\hline
\end{tabular}

Compared to patients in the normal renal function category [mean age $(95 \% \mathrm{Cl}) 42.66(42.03,43.30)$ years], those in the mildly impaired and moderately/severely impaired renal function categories were significantly older $[52.59(51.74,53.43)$ years; $58.62(54.59,62.65)$ years; both $P=0.0000)$. Also, the mean values for BMI, fasting and 2-h post prandial glucose, SBP, and DBP, as well as the estimated prevalence rates of overweight/obesity, prediabetes and diabetes, and hypertension, showed significantly increasing trends with decreasing eGFR categories, whereas the estimated prevalence of underweight was decreased in participants with mildly impaired eGFR (Table 1).

Notably, significant increases in TG, TC, and LDL-C were accompanied by decreases in eGFR categories. In the normal, mildly impaired, and moderately/severely impaired eGFR groups, the mean TG levels were 1.48, 1.73, and $1.94 \mathrm{mmol} / \mathrm{L}$, respectively; the mean TC levels were $4.67,4.81$, and $5.07 \mathrm{mmol} / \mathrm{L}$, respectively; and the mean LDL-C levels were 2.72, 2.84, and $3.08 \mathrm{mmol} / \mathrm{L}$, respectively (compared to the normal eGFR category, all $P<0.05$ ). For HDL-C, no significant difference was observed among eGFR categories (Table 1).

In addition, the mean UACR was significantly higher in the moderately/severely impaired eGFR group [mean $(95 \% \mathrm{Cl}) 98.35(64.01,132.70) \mathrm{mg} / \mathrm{g}$ ] than in the normal eGFR group $[27.23(25.14,29.31) \mathrm{mg} / \mathrm{g}, P=0.0000]$ or the mildly impaired eGFR group $[32.19(28.34,36.03) \mathrm{mg} / \mathrm{g}, P=0.0000](\mathrm{Table} 1)$.

Mean values of eGFR and estimated prevalence rates of eGFR impairment according to categories of TC, TG, HDL-C, and LDL-C

When the mean value of eGFR was calculated and compared between categories, a significantly lower eGFR was observed only for the borderline high TG [1.7 $\sim 2.3 \mathrm{mmol} / \mathrm{L}: 110.99(105.44,116.55) \mathrm{ml} / \mathrm{min}$ per $\left.1.73 \mathrm{~m}^{2}, P=0.0000\right]$ and the high TG categories $[\geq 2.3 \mathrm{mmol} / \mathrm{L}: 111.62(107.85,115.39) \mathrm{ml} / \mathrm{min} \mathrm{per}$ $\left.1.73 \mathrm{~m}^{2}, P=0.0000\right]$ compared with the normal TG category $\left[<1.7 \mathrm{mmol} / \mathrm{L}: 122.03(117.00,127.07) \mathrm{ml} / \mathrm{min}\right.$ per $\left.1.73 \mathrm{~m}^{2}\right]$. Also, the estimated prevalence of mildly impaired eGFR was significantly higher for the borderline high TG $(30.92 \%, P=0.0000)$ and high TG categories $(27.92 \%, P=0.0000)$ compared with the normal TG category (19.96\%), and the estimated prevalence of moderately/severely impaired eGFR for the high TG category (2.81\%) was significantly higher than that for the normal TG category $(1.20 \%, P=0.0174)$. Also, a higher prevalence of mildly impaired eGFR was observed with $3.4 \mathrm{mmol} / \mathrm{L} \leq \mathrm{LDL}-\mathrm{C}<$ 
$4.1 \mathrm{mmol} / \mathrm{L}(27.93 \%)$ compared with LDL-C $<3.4 \mathrm{mmol} / \mathrm{L}(22.61 \%, P=0.0092)$. For the other comparisons, no statistically significant differences were observed (Table 2).

\title{
Associations of TC, TG, HDL-C, LDL-C, and their categories with eGFR
}

As shown in Table 3, after adjustment for the conventional confounding factors including age, gender, BMI, smoking, alcohol intake habit, diabetes, and hypertension, increased TG and decreased HDL-C levels showed independently significant associations with reduced eGFR [TG: $\beta($ SE) -0.0238 (0.0039), $P=$ 0.0000; HDL-C (decreased): -0.0883 (0.0176), $P=0.0000]$, whereas elevated TC and LDL-C levels were associated with an increased eGFR [TC: 0.0314 (0.0060), $P=0.0000$; LDL-C: 0.0251 (0.0070), $P=0.0004]$

Table 3

Associations of total cholesterol, triglycerides, high-density lipoprotein-cholesterol, low-density lipoprotein-cholesterol or their categories with the estimated glomerular filtration rate and the risks for estimated glomerular filtration rate impairment.

\begin{tabular}{|c|c|c|c|c|c|c|}
\hline \multirow{2}{*}{$\begin{array}{l}\text { Model } \\
\text { Outcome }\end{array}$} & \multicolumn{2}{|l|}{ Regression model } & \multicolumn{4}{|c|}{ Multinomial logistic model } \\
\hline & \multicolumn{2}{|l|}{ Ln eGFR } & \multicolumn{2}{|c|}{$\begin{array}{l}\text { Mildly impaired eGFR } \\
\text { (eGFR } 60 \sim 90 \mathrm{ml} / \mathrm{min} \text { per } 1.73 \mathrm{~m}^{2} \text { ) }\end{array}$} & \multicolumn{2}{|c|}{$\begin{array}{l}\text { Moderately/severely impaired eGFF } \\
\left(\text { eGFR }<60 \mathrm{ml} / \mathrm{min} \text { per } 1.73 \mathrm{~m}^{2} \text { ) }\right.\end{array}$} \\
\hline Independent variable & $\boldsymbol{\beta}$ (SE) & $\mathrm{P}$ & OR $(95 \% \mathrm{Cl})$ & $\mathrm{P}$ & OR $(95 \% \mathrm{Cl})$ & $\mathrm{P}$ \\
\hline TC, per $1 \mathrm{mmol} / \mathrm{L}$ increase & $0.0314(0.0060)$ & 0.0000 & $0.91(0.84,0.99)$ & 0.0302 & $1.05(0.74,1.49)$ & 0.7799 \\
\hline \multicolumn{7}{|l|}{ TC categories } \\
\hline Normal: $<5.2 \mathrm{mmol} / \mathrm{L}$ & As reference & - & As reference & - & As reference & - \\
\hline Borderline high: $5.2 \sim 6.3 \mathrm{mmol} / \mathrm{L}$ & $0.0504(0.0114)$ & 0.0000 & $0.82(0.67,1.02)$ & 0.0713 & $0.80(0.42,1.51)$ & 0.4926 \\
\hline High: $\geq 6.3 \mathrm{mmol} / \mathrm{L}$ & $0.0477(0.0358)$ & 0.1822 & $0.82(0.62,1.10)$ & 0.1836 & $2.18(0.76,6.23)$ & 0.1450 \\
\hline TG, per $1 \mathrm{mmol} / \mathrm{L}$ increase & $-0.0238(0.0039)$ & 0.0000 & $1.19(1.12,1.26)$ & 0.0000 & $1.31(1.15,1.50)$ & 0.0001 \\
\hline
\end{tabular}

TG categories

Normal: $<1.7 \mathrm{mmol} / \mathrm{L}$

As reference

Borderline high: $1.7 \sim 2.3 \mathrm{mmol} / \mathrm{L}$

$-0.0674(0.0148)$

- $\quad$ As reference

-0.0674 (0.0139)

0.0000

$1.69(1.38,2.08)$

$0.0000 \quad 1.65(1.34,2.02)$

$-0.0883(0.0176)$

0.0000

$0.93(0.87,1.33)$

$-$

High: $\geq 2.3 \mathrm{mmol} / \mathrm{L}$

HDL-C, per $1 \mathrm{mmol} / \mathrm{L}$ decrease

HDL-C categories

Normal: $\geq 1.0 \mathrm{mmol} / \mathrm{L}$

As reference

Low: $<1.0 \mathrm{mmol} / \mathrm{L}$

$-0.0240(0.0122)$

- $\quad$ As reference

$0.0251(0.0070)$

0.0497

$0.96(0.78,1.19)$

$0.0004 \quad 0.91(0.82,1.01)$

0.0689

As reference

0.0000

$1.83(0.85,3.97)$

$-$

LDL-C, per $1 \mathrm{mmol} / \mathrm{L}$ increase

$0.0251(0.0070)$

0.0004

$9.91(0.82,1.01)$

- $\quad$ As reference

Normal: $<3.4 \mathrm{mmol} / \mathrm{L}$

Borderline high: $3.4 \sim 4.1 \mathrm{mmol} / \mathrm{L}$

\begin{abstract}
As reference
\end{abstract}
0.0246 (0.0178)

0.1655

$0.93(0.73,1.20)$

$0.04040 .62(0.40,0.96)$

$0.0597(0.0291)$

High: $\geq 4.1 \mathrm{mmol} / \mathrm{L}$

Abbreviations: BMI, body mass index; $\mathrm{Cl}$, confidence interval; eGFR, estimated glomerular filtration rate; HDL-C, high-density lipoprotein-cholesterol; LDL-C, low-density lipoprotein-cholesterol; OR, odds ratio; SE, standard error; TC, total cholesterol; TG, triglycerides.

All calculations were weighted based on the Chinese population data and the study sampling scheme. Regression models or multinomial logistic models were used to test the impact of TG, TC, HDL-C, and LDL-C and their categories on eGFR or the risks for mildly impaired eGFR $\left(60 \sim 90 \mathrm{ml} / \mathrm{min}^{\mathrm{per}} 1.73 \mathrm{~m}{ }^{2}\right)$ and moderately/severely impaired eGFR $\left(<60 \mathrm{ml} / \mathrm{min}\right.$ per $\left.1.73 \mathrm{~m}^{2}\right)$, respectively. EGFR were natural logarithmically transformed prior to the regression. Age, gender, BMl, smoking status, alcohol intake status, diabetes status, and hypertension status were adjusted as confounding factors in these models. $P$ values $<0.05$ are shown in bold.

Upon stratification of the four measurements into categories, both the borderline high [1.7 2.3 mmol/L: $\beta(\mathrm{SE})-0.0674(0.0148), P=0.0000]$ and high TG categories [ $\geq 2.3 \mathrm{mmol} / \mathrm{L}:-0.0674(0.0139), P=0.0000$ ] as well as the low HDL-C category [ $1.0 \mathrm{mmol} / \mathrm{L}:-0.0240(0.0122), P=0.0497]$ were significantly associated with a decreased eGFR. In contrast, the borderline high TC [5.2 $6.3 \mathrm{mmol} / \mathrm{L}: 0.0504(0.0114), P=0.0000]$ and the high LDL-C categories $[\geq$ $4.1 \mathrm{mmol} / \mathrm{L}: 0.0597$ (0.0291), $P=0.0404]$ were related to an increased eGFR (Table 3).

\section{Associations of TC, TG, HDL-C, LDL-C, and their categories with the risks for eGFR impairment}

Using multinomial logistic analyses with adjustment for the conventional risk factors, we identified that each per mmol/L increase in TG increased the risks for mildly and moderately to severely impaired eGFR with ORs and $95 \%$ Cls of $1.19(1.12,1.26)(P=0.0000)$ and $1.31(1.15,1.50)(P=0.0001)$. Each per 
$\mathrm{mmol} / \mathrm{L}$ increase in TC was associated with a decreased risk for mildly impaired eGFR [OR $(95 \% \mathrm{Cl}) 0.91(0.84,0.99), P=0.0302]$. However, neither HDL-C nor LDL-C was related to the risks for eGFR impairment (all $P>0.05$; Table 3).

Moreover, in contrast to the normal TG category $(<1.7 \mathrm{mmol} / \mathrm{L})$, the borderline high TG and high TG categories were significantly associated with 1.69 -fold and 1.65-fold increased risks for mildly impaired eGFR $(P=0.0000,0.0000)$, respectively, and the high TG category was associated with a 2.52 -fold increase in the risk for moderately/severely impaired eGFR $(P=0.0209)$. The high LDL-C category was associated with a reduced risk for mildly impaired eGFR by $0.62-$ fold $(P=0.0319 ;$ Table 3).

\section{Associations of $T C, T G, H D L-C$, and $L D L-C$ with eGFR in subpopulations stratified by conventional risk factors}

We identified significant interactions between HDL-C and gender $\left(P_{H D L-C^{*} \text { gender }}=0.0000\right)$, BMI category $\left(P_{H D L-C * B M I}\right.$ category $\left.=0.0292\right)$, smoking status $\left(P_{H D L-C *}{ }^{\text {smoking status }}=0.0260\right)$, and alcohol intake habit $\left(P_{H D L-C * a l c o h o l ~ i n t a k e}=0.0001\right)$ for the effect on eGFR. When the overall population was further stratified, significantly larger absolute effective sizes upon eGFR were identified in males [ $\beta$ (SE) $-0.1556(0.0246), P=0.0000]$ versus females $[-0.0288(0.0233), P=$ $0.2160]$, in overweight/obese [-0.1329 (0.0246), $P=0.0000]$ versus underweight $[-0.0772(0.0478), P=0.1060]$ or normal weight individuals $[-0.0559(0.0247), P$ $=0.0236]$, in smokers [-0.1380 (0.0340), $P=0.0000]$ versus non-smokers [-0.0673 (0.0202), $P=0.0009]$, and with alcohol intake habit $[-0.1817(0.0339), P=$ $0.0000]$ versus without $[-0.0449(0.0203), P=0.0272]$. However, no significant interactions of TC, TG, or LDL-C with any of the conventional risk factors for the effect on eGFR were observed (all $P>0.05$; Table 4). 
Table 4

Associations of total cholesterol, triglycerides, high-density lipoprotein-cholesterol, and low-density lipoprotein-cholesterol with the estimated glomerular filtration rate in the stratified populations.

\begin{tabular}{|c|c|c|c|c|c|c|c|c|}
\hline \multirow[t]{2}{*}{ Subpopulation } & \multicolumn{2}{|c|}{ TC, per $1 \mathrm{mmol} / \mathrm{L}$ increase } & \multicolumn{2}{|c|}{ TG, per $1 \mathrm{mmol} / \mathrm{L}$ increase } & \multicolumn{2}{|c|}{ HDL-C, per $1 \mathrm{mmol} / \mathrm{L}$ decrease } & \multicolumn{2}{|c|}{ LDL-C, per $1 \mathrm{mmol} / \mathrm{L}$ increase } \\
\hline & $\beta(\mathrm{SE})$ & $\mathbf{P}$ & $\beta(\mathrm{SE})$ & $\mathbf{P}$ & $\beta(\mathrm{SE})$ & $\mathbf{P}$ & $\beta(\mathrm{SE})$ & $\mathbf{P}$ \\
\hline Gender & \multicolumn{8}{|c|}{ (Age, BMI, smoking status, alcohol intake status, diabetes status, hypertension status, and TC/TG/HDL-C/LDL-C) } \\
\hline Males & $0.0339(0.0078)$ & 0.0000 & $-0.0210(0.0051)$ & 0.0000 & $-0.1556(0.0246)$ & 0.0000 & $0.0311(0.0099)$ & 0.0017 \\
\hline Females & $0.0313(0.0085)$ & 0.0002 & $-0.0255(0.0062)$ & 0.0000 & $-0.0288(0.0233)$ & 0.2160 & $0.0205(0.0095)$ & 0.0302 \\
\hline \multirow[t]{2}{*}{$P_{\text {Gender interaction }}$} & \multicolumn{8}{|c|}{$\begin{array}{l}\text { (Age, gender, BMl, smoking status, alcohol intake status, diabetes status, hypertension status, TC/TG/HDL-C/LDL-C, and } \\
\text { TC/TG/HDL-C/LDL-C*gender) }\end{array}$} \\
\hline & \multicolumn{2}{|l|}{$P_{T C^{\star} \text { gender }}=0.8371$} & \multicolumn{2}{|l|}{$P_{T G^{*} \text { gender }}=0.9236$} & \multicolumn{2}{|c|}{$P_{H D L-C^{*} \text { gender }}=0.0000$} & \multicolumn{2}{|c|}{$P_{L D L^{-} C^{*} \text { gender }}=0.7402$} \\
\hline Age categories & \multicolumn{8}{|c|}{ (Age, gender, BMI, smoking status, alcohol intake status, diabetes status, hypertension status, and TC/TG/HDL-C/LDL-C) } \\
\hline$<40$ years & $0.0343(0.0091)$ & 0.0002 & $-0.0192(0.0066)$ & 0.0037 & $-0.1003(0.0283)$ & 0.0004 & $0.0224(0.0126)$ & 0.0764 \\
\hline $40 \sim 60$ years & $0.0363(0.0069)$ & 0.0000 & $-0.0237(0.0054)$ & 0.0000 & $-0.0849(0.0210)$ & 0.0001 & $0.0382(0.0076)$ & 0.0000 \\
\hline$\geq 60$ years & $0.0202(0.0187)$ & 0.2797 & $-0.0291(0.0119)$ & 0.0143 & $-0.0655(0.0574)$ & 0.2537 & $0.0047(0.0197)$ & 0.8123 \\
\hline \multirow[t]{2}{*}{$\mathrm{P}_{\text {Age interaction }}$} & \multicolumn{8}{|c|}{$\begin{array}{l}\text { (Age category, gender, BMl, smoking status, alcohol intake status, diabetes status, hypertension status, TC/TG/HDL-C/LDL-C, anc } \\
\text { TC/TG/HDL-C/LDL-C*age category) }\end{array}$} \\
\hline & \multicolumn{2}{|c|}{$P_{T C{ }^{*} \text { age category }}=0.6567$} & \multicolumn{2}{|c|}{$P_{T G^{*} \text { age category }}=0.5546$} & \multicolumn{2}{|c|}{$P_{H D L-C^{*} \text { age category }}=0.5610$} & \multicolumn{2}{|c|}{$P_{L D L-C * a g e ~ c a t e g o r y}=0.3025$} \\
\hline BMI categories & \multicolumn{8}{|c|}{ (Age, gender, BMI, smoking status, alcohol intake status, diabetes status, hypertension status, and TC/TG/HDL-C/LDL-C) } \\
\hline$<18.5 \mathrm{~kg} / \mathrm{m}^{2}$ & $0.0541(0.0177)$ & 0.0022 & $0.0115(0.0399)$ & 0.7729 & $-0.0772(0.0478)$ & 0.1060 & $0.0286(0.0203)$ & 0.1591 \\
\hline $18.5 \sim 24 \mathrm{~kg} / \mathrm{m}^{2}$ & $0.0261(0.0093)$ & 0.0051 & $-0.0241(0.0080)$ & 0.0027 & $-0.0559(0.0247)$ & 0.0236 & $0.0091(0.0104)$ & 0.3790 \\
\hline$\geq 24 \mathrm{~kg} / \mathrm{m}^{2}$ & $0.0379(0.0075)$ & 0.0000 & $-0.0217(0.0043)$ & 0.0000 & $-0.1329(0.0246)$ & 0.0000 & $0.0422(0.0099)$ & 0.0000 \\
\hline \multirow[t]{2}{*}{$\mathrm{P}_{\mathrm{BMl} \text { interaction }}$} & \multicolumn{8}{|c|}{$\begin{array}{l}\text { (Age, gender, BMI category, smoking status, alcohol intake status, diabetes status, hypertension status, TC/TG/HDL-C/LDL-C, anc } \\
\text { TC/TG/HDL-C/LDL-C*BMl category) }\end{array}$} \\
\hline & \multicolumn{2}{|c|}{$P_{T C * B M I}$ category $=0.4094$} & \multicolumn{2}{|c|}{$P_{T G^{*} B M I}$ category $=0.6485$} & \multicolumn{2}{|c|}{$P_{H D L-C * B M I}$ category $=0.0292$} & \multicolumn{2}{|c|}{$P_{L D L-C * B M I}$ category $=0.0811$} \\
\hline Smoking status & \multicolumn{8}{|c|}{ (Age, gender, BMI, alcohol intake status, diabetes status, hypertension status, and TC/TG/HDL-C/LDL-C) } \\
\hline No & $0.0296(0.0071)$ & 0.0000 & $-0.0280(0.0050)$ & 0.0000 & $-0.0673(0.0202)$ & 0.0009 & $0.0226(0.0084)$ & 0.0073 \\
\hline Yes & $0.0397(0.0102)$ & 0.0001 & $-0.0148(0.0061)$ & 0.0151 & $-0.1380(0.0340)$ & 0.0000 & $0.0362(0.0115)$ & 0.0017 \\
\hline$P_{\text {Smoking status interaction }}$ & $\begin{array}{l}\text { (Age, gender, BM } \\
\text { TC/TG/HDL-C/L }\end{array}$ & $\begin{array}{l}\text { noking s } \\
C^{\star} \text { smoki }\end{array}$ & $\begin{array}{l}\text { atus, alcohol intak } \\
\text { g status) }\end{array}$ & atus, di & es status, hyper & status & TG/HDL-C/LDL & \\
\hline & $P_{T C *_{\text {Smoking status }}}=$ & 7628 & $P_{T G * \text { smoking status }}=$ & 2760 & $P_{H D L-C \star^{*} \text { smoking sta }}$ & .0260 & $P_{L D L-C^{*} \text { smoking st }}$ & 7070 \\
\hline Alcohol intake status & (Age, gender, BMI, & moking s & atus, diabetes statu & hyperten & on status, and $\mathrm{TC} / \mathrm{T}$ & HDL-C/LD & & \\
\hline No & $0.0249(0.0068)$ & 0.0003 & $-0.0261(0.0047)$ & 0.0000 & $-0.0449(0.0203)$ & 0.0272 & $0.0253(0.0079)$ & 0.0013 \\
\hline Yes & $0.0502(0.0118)$ & 0.0000 & $-0.0175(0.0068)$ & 0.0104 & $-0.1817(0.0339)$ & 0.0000 & $0.0249(0.0142)$ & 0.0798 \\
\hline$P_{\text {Alcohol intake interaction }}$ & $\begin{array}{l}\text { (Age, gender, BMI } \\
\text { TC/TG/HDL-C/LL }\end{array}$ & $\begin{array}{l}\text { hoking s } \\
*^{*} \text { alcohe }\end{array}$ & $\begin{array}{l}\text { tus, alcohol intak } \\
\text { intake status) }\end{array}$ & atus, di & es status, hyper & statu & TG/HDL-C/LD & \\
\hline & $P_{T C * a / c o h o l ~ i n t a k e}=0$ & 0831 & $P_{T G * a l c o h o l ~ i n t a k e}=0$ & 387 & 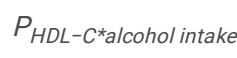 & 001 & $P_{L D L-C^{*} \text { alcohol intake }}$ & 9220 \\
\hline Diabetes status & (Age, gender, BMI & moking s & atus, alcohol intake & tatus, hyp & rtension status, a & /TG/HD & /LDL-C) & \\
\hline $\begin{array}{l}\text { Normal glycemic } \\
\text { metabolism }\end{array}$ & $0.0391(0.0064)$ & 0.0000 & $-0.0254(0.0054)$ & 0.0000 & $-0.0973(0.0181)$ & 0.0000 & $0.0295(0.0082)$ & 0.0003 \\
\hline Prediabetes & $0.0224(0.0137)$ & 0.1027 & $-0.0135(0.0071)$ & 0.0586 & $-0.0930(0.0333)$ & 0.0052 & $0.0243(0.0144)$ & 0.0908 \\
\hline Diabetes & $-0.0020(0.0224)$ & 0.9298 & $-0.0305(0.0086)$ & 0.0004 & 0.0075 (0.0965) & 0.9377 & $-0.0013(0.0211)$ & 0.9497 \\
\hline breviations: BMI, bc & $\begin{array}{l}\text { nass index; BP, } \\
\text { sity lipoprotein }\end{array}$ & $\begin{array}{l}\text { press } \\
\text { esterol }\end{array}$ & $\begin{array}{l}\mathrm{Cl} \text {, confidence in } \\
\text {, standard error; }\end{array}$ & $\begin{array}{l}\text { al; eGF } \\
\text { otal ch }\end{array}$ & $\begin{array}{l}\text { timated glomer } \\
\text { terol; TG, triglyc }\end{array}$ & ation & DL-C, high-der & protein- \\
\hline $\begin{array}{l}\text { All calculations were w } \\
\text { TC, HDL-C, or LDL-C on } \\
\text { status, separately. In th } \\
\text { logarithmically transfor } \\
\text { shown in bold. }\end{array}$ & $\begin{array}{l}\text { ted based on C } \\
\text { FR in subpopula } \\
\text { verall cohort, the } \\
\text { d prior to the re }\end{array}$ & $\begin{array}{l}\text { se popu } \\
\text { is stratif } \\
\text { eraction }\end{array}$ & $\begin{array}{l}\text { tion data and the } \\
\text { by gender, age, } \\
\text { rms were further } \mathrm{i} \\
\text { bles included in } \mathrm{t}\end{array}$ & $\begin{array}{l}\text { dy samp } \\
\text { smokin } \\
\text { aded res } \\
\text { egressio }\end{array}$ & $\begin{array}{l}\text { g scheme. Regre } \\
\text { tatus, alcohol int } \\
\text { tively to examin } \\
\text { nodels were den }\end{array}$ & $\begin{array}{l}\text { nodels } \\
\text { atus, di } \\
\text { hteracti } \\
\text { the pa }\end{array}$ & $\begin{array}{l}\text { used to test the } \\
\text { es status, and hy } \\
\text { ffects. EGFR wer } \\
\text { leses. } P \text { values < }\end{array}$ & $\begin{array}{l}\text { ct of TG, } \\
\text { nsion } \\
\text { ural } \\
\text { are }\end{array}$ \\
\hline
\end{tabular}




\begin{tabular}{|c|c|c|c|c|c|c|c|c|}
\hline \multirow[t]{2}{*}{ Subpopulation } & \multicolumn{2}{|c|}{ TC, per $1 \mathrm{mmol} / \mathrm{L}$ increase } & \multicolumn{2}{|c|}{ TG, per $1 \mathrm{mmol} / \mathrm{L}$ increase } & \multicolumn{2}{|c|}{ HDL-C, per $1 \mathrm{mmol} / \mathrm{L}$ decrease } & \multicolumn{2}{|c|}{ LDL-C, per $1 \mathrm{mmol} / \mathrm{L}$ increase } \\
\hline & $\beta(\mathrm{SE})$ & $\mathbf{P}$ & $\beta(S E)$ & $\mathbf{P}$ & $\beta(S E)$ & $\mathbf{P}$ & $\beta$ (SE) & $\mathbf{P}$ \\
\hline \multirow[t]{2}{*}{$P_{\text {Diabetes status interaction }}$} & \multicolumn{8}{|c|}{$\begin{array}{l}\text { (Age, gender, BMl, smoking status, alcohol intake status, diabetes status, hypertension status, TC/TG/HDL-C/LDL-C, and } \\
\text { TC/TG/HDL-C/LDL-C^diabetes status) }\end{array}$} \\
\hline & \multicolumn{2}{|c|}{$P_{T C * \text { diabetes status }}=0.2330$} & \multicolumn{2}{|c|}{$P_{T G^{*} \text { diabetes status }}=0.2828$} & \multicolumn{2}{|c|}{$P_{H D L-C C^{*} \text { diabetes status }}=0.3300$} & \multicolumn{2}{|c|}{$P_{L D L-C * \text { diabetes status }}=0.3028$} \\
\hline Hypertension status & \multicolumn{8}{|c|}{ (Age, gender, BMI, smoking status, alcohol intake status, diabetes status, and TC/TG/HDL-C/LDL-C) } \\
\hline Normal tension & $0.0255(0.0097)$ & 0.0087 & $-0.0288(0.0086)$ & 0.0008 & $-0.0718(0.0259)$ & 0.0056 & $0.0107(0.0118)$ & 0.3614 \\
\hline Prehypertension & $0.0364(0.0079)$ & 0.0000 & $-0.0220(0.0051)$ & 0.0000 & $-0.0982(0.0238)$ & 0.0000 & $0.0341(0.0108)$ & 0.0016 \\
\hline Hypertension & $0.0345(0.0140)$ & 0.0141 & $-0.0221(0.0075)$ & 0.0034 & $-0.1006(0.0474)$ & 0.0336 & $0.0319(0.0139)$ & 0.0217 \\
\hline \multirow[t]{2}{*}{$\begin{array}{l}\text { P Hypertension status } \\
\text { interaction }\end{array}$} & \multicolumn{8}{|c|}{$\begin{array}{l}\text { (Age, gender, BMl, smoking status, alcohol intake status, diabetes status, hypertension status, TC/TG/HDL-C/LDL-C, and } \\
\text { TC/TG/HDL-C/LDL-C* hypertension status) }\end{array}$} \\
\hline & \multicolumn{2}{|c|}{$\begin{array}{l}P_{T C * \text { hypertension status }} \\
=0.8402\end{array}$} & \multicolumn{2}{|l|}{$\begin{array}{l}P_{\text {TG*hypertension status }} \\
=0.8852\end{array}$} & \multicolumn{2}{|c|}{ 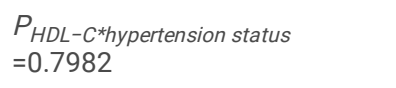 } & \multicolumn{2}{|c|}{$P_{L D L-C * \text { hypertension status }}=0.4096$} \\
\hline \multicolumn{9}{|c|}{$\begin{array}{l}\text { Abbreviations: BMI, body mass index; BP, blood pressure; Cl, confidence interval; eGFR, estimated glomerular filtration rate; HDL-C, high-density lipoprotein- } \\
\text { cholesterol; LDL-C, low-density lipoprotein-cholesterol; SE, standard error; TC, total cholesterol; TG, triglycerides. }\end{array}$} \\
\hline \multicolumn{9}{|c|}{$\begin{array}{l}\text { All calculations were weighted based on Chinese population data and the study sampling scheme. Regression models were used to test the impact of TG, } \\
\text { TC, HDL-C, or LDL-C on eGFR in subpopulations stratified by gender, age, BMI, smoking status, alcohol intake status, diabetes status, and hypertension } \\
\text { status, separately. In the overall cohort, the interaction terms were further included respectively to examine the interaction effects. EGFR were natural } \\
\text { logarithmically transformed prior to the regression. Variables included in the regression models were denoted in the parentheses. } P \text { values }<0.05 \text { are } \\
\text { shown in bold. }\end{array}$} \\
\hline
\end{tabular}

\section{Associations of TC, TG, HDL-C, and LDL-C with the risks for eGFR impairment in subpopulations stratified by conventional risk factors}

TG was found to interact with gender in affecting the risk for eGFR impairment $\left(P_{T G^{*} \text { gender }}=0.0261\right)$. Each per mmol/L increase in TG was significantly associated with 1.16 -fold and 1.27 -fold increases in the risk for mildly impaired eGFR in males and females, respectively $(P=0.0002,0.0000)$. Notably, each per $\mathrm{mmol} / \mathrm{L}$ increase in TG increased the risk for moderately/severely impaired eGFR in males only (by 1.45 -fold, $P=0.0000)$ and not in females $(P=0.1185$; Table 5). 
Table 5

Associations of total cholesterol, triglycerides, high-density lipoprotein-cholesterol, and low-density lipoprotein-cholesterol with the risks for estimated glomerul

\begin{tabular}{|c|c|c|c|c|c|c|c|c|c|c|c|c|c|}
\hline \multirow{5}{*}{ Subpopulation } & \multirow{2}{*}{\multicolumn{4}{|c|}{ TC, per $1 \mathrm{mmol} / \mathrm{L}$ increase }} & \multirow{2}{*}{\multicolumn{4}{|c|}{$T G, p$}} & \multirow{2}{*}{\multicolumn{4}{|c|}{ HDL-C, per $1 \mathrm{mmol} / \mathrm{L}$ decrease }} & \\
\hline & & & & & & & & & & & & & l \\
\hline & \multirow{2}{*}{\multicolumn{2}{|c|}{$\begin{array}{l}\text { Mildly impaired } \\
\text { eGFR } \\
(\text { eGFR } 60 \sim \\
90 \mathrm{ml} / \mathrm{min} \text { per } \\
1.73 \mathrm{~m}^{2} \text { ) }\end{array}$}} & \multirow{2}{*}{\multicolumn{2}{|c|}{$\begin{array}{l}\text { Moderately/severely } \\
\text { impaired eGFR } \\
(\text { eGFR }<60 \mathrm{ml} / \mathrm{min} \\
\text { per } 1.73 \mathrm{~m}^{2} \text { ) }\end{array}$}} & \multirow{2}{*}{\multicolumn{2}{|c|}{$\begin{array}{l}\text { Mildly impaired } \\
\text { eGFR } \\
\text { (eGFR 60 } \\
90 \mathrm{ml} / \mathrm{min} \text { per } \\
1.73 \mathrm{~m}^{2} \text { ) }\end{array}$}} & \multirow{2}{*}{\multicolumn{2}{|c|}{$\begin{array}{l}\text { Moderately/severely } \\
\text { impaired eGFR } \\
\text { (eGFR } 600 \mathrm{ml} / \mathrm{min} \\
\text { per } 1.73 \mathrm{~m}^{2} \text { ) }\end{array}$}} & \multirow{2}{*}{\multicolumn{2}{|c|}{$\begin{array}{l}\text { Mildly impaired } \\
\text { eGFR } \\
(\text { eGFR } 60 \sim \\
90 \mathrm{ml} / \mathrm{min} \text { per } \\
1.73 \mathrm{~m}^{2} \text { ) }\end{array}$}} & \multirow{2}{*}{\multicolumn{2}{|c|}{$\begin{array}{l}\text { Moderately/severely } \\
\text { impaired eGFR } \\
(\text { eGFR }<60 \mathrm{ml} / \mathrm{min} \\
\text { per } 1.73 \mathrm{~m}^{2} \text { ) }\end{array}$}} & \\
\hline & & & & & & & & & & & & & \\
\hline & $\begin{array}{l}\text { OR } \\
(95 \% \mathrm{Cl})\end{array}$ & $\mathbf{P}$ & $\begin{array}{l}\text { OR } \\
(95 \% \mathrm{Cl})\end{array}$ & $\mathbf{P}$ & $\begin{array}{l}\text { OR } \\
(95 \% \mathrm{Cl})\end{array}$ & $\mathbf{P}$ & $\begin{array}{l}\text { OR } \\
(95 \% \mathrm{Cl})\end{array}$ & $\mathbf{P}$ & $\begin{array}{l}\text { OR } \\
(95 \% \mathrm{Cl})\end{array}$ & $\mathbf{P}$ & $\begin{array}{l}\text { OR } \\
(95 \% \mathrm{Cl})\end{array}$ & $\mathbf{P}$ & \\
\hline Gender & \multicolumn{13}{|c|}{ (Age, BMI, smoking status, alcohol intake status, diabetes status, hypertension status, and TC/TG/HDL-C/LDL-C) } \\
\hline Males & $\begin{array}{l}0.93 \\
(0.82 \\
1.06)\end{array}$ & 0.2801 & $\begin{array}{l}0.95 \\
(0.56 \\
1.6)\end{array}$ & 0.8526 & $\begin{array}{l}1.16 \\
(1.07 \\
1.25)\end{array}$ & 0.0002 & $\begin{array}{l}1.45 \\
(1.24, \\
1.69)\end{array}$ & 0.0000 & $\begin{array}{l}1.23 \\
(0.84 \\
1.79)\end{array}$ & 0.2834 & $\begin{array}{l}20.00 \\
(4.76 \\
100.00)\end{array}$ & 0.0001 & \\
\hline Females & $\begin{array}{l}0.93 \\
(0.83 \\
1.03)\end{array}$ & 0.1577 & $\begin{array}{l}1.13 \\
(0.73 \\
1.75)\end{array}$ & 0.5788 & $\begin{array}{l}1.27 \\
(1.15 \\
1.39)\end{array}$ & 0.0000 & $\begin{array}{l}1.17 \\
(0.96 \\
1.43)\end{array}$ & 0.1185 & $\begin{array}{l}0.98 \\
(0.76 \\
1.27)\end{array}$ & 0.8681 & $\begin{array}{l}0.53 \\
(0.17 \\
1.69)\end{array}$ & 0.2886 & \\
\hline $\mathrm{P}_{\text {Gender interaction }}$ & \multicolumn{13}{|c|}{ (Age, gender, BMI, smoking status, alcohol intake status, diabetes status, hypertension status, TC/TG/HDL-C/LDL-C, and TC/TG/HDL-C/L } \\
\hline & \multicolumn{4}{|c|}{$P_{T C * \text { gender }}=0.4602$} & \multicolumn{4}{|c|}{$P_{T G^{*} \text { gender }}=\mathbf{0 . 0 2 6 1}$} & \multicolumn{4}{|c|}{$P_{H D L-C^{*} \text { gender }}=0.0003$} & $t$ \\
\hline Age categories & \multicolumn{13}{|c|}{ (Age, gender, BMI, smoking status, alcohol intake status, diabetes status, hypertension status, and TC/TG/HDL-C/LDL-C) } \\
\hline$<40$ years & $\begin{array}{l}1.00 \\
(0.87 \\
1.16)\end{array}$ & 0.9489 & $\begin{array}{l}1.12 \\
(0.47 \\
2.68)\end{array}$ & 0.8003 & $\begin{array}{l}1.09 \\
(0.96 \\
1.24)\end{array}$ & 0.1753 & $\begin{array}{l}1.07 \\
(0.79 \\
1.46)\end{array}$ & 0.6709 & $\begin{array}{l}0.76 \\
(0.52 \\
1.12)\end{array}$ & 0.1703 & $\begin{array}{l}0.54 \\
(0.06 \\
5.26)\end{array}$ & 0.5913 & \\
\hline $40 \sim 60$ years & $\begin{array}{l}0.88 \\
(0.79 \\
0.97)\end{array}$ & 0.0137 & $\begin{array}{l}0.79 \\
(0.50 \\
1.24)\end{array}$ & 0.3085 & $\begin{array}{l}1.19 \\
(1.11, \\
1.28)\end{array}$ & 0.0000 & $\begin{array}{l}1.47 \\
(1.25, \\
1.73)\end{array}$ & 0.0000 & $\begin{array}{l}1.28 \\
(0.97 \\
1.67)\end{array}$ & 0.0793 & $\begin{array}{l}2.22 \\
(0.66 \\
7.14)\end{array}$ & 0.2001 & \\
\hline$\geq 60$ years & $\begin{array}{l}0.89 \\
(0.74 \\
1.07)\end{array}$ & 0.2137 & $\begin{array}{l}1.19 \\
(0.73 \\
1.95)\end{array}$ & 0.4903 & $\begin{array}{l}1.28 \\
(1.09 \\
1.51)\end{array}$ & 0.0032 & $\begin{array}{l}1.30 \\
(0.99 \\
1.71)\end{array}$ & 0.0577 & $\begin{array}{l}1.08 \\
(0.65 \\
1.79)\end{array}$ & 0.7701 & $\begin{array}{l}1.14 \\
(0.15 \\
8.33)\end{array}$ & 0.9057 & \\
\hline
\end{tabular}

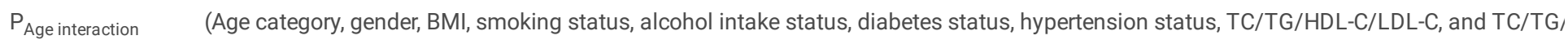

$$
P_{T C^{\star} \text { age category }}=0.1278 \quad P_{T G^{\star} \text { age category }}=0.6197 \quad P_{H D L-C^{*} \text { age category }}=0.6775
$$

BMl categories (Age, gender, BMl, smoking status, alcohol intake status, diabetes status, hypertension status, and TC/TG/HDL-C/LDL-C)

\begin{tabular}{|c|c|c|c|c|c|c|c|c|c|c|c|c|}
\hline$<18.5 \mathrm{~kg} / \mathrm{m}^{2}$ & $\begin{array}{l}0.62 \\
(0.37 \\
1.03)\end{array}$ & 0.0653 & $\begin{array}{l}0.53 \\
(0.22 \\
1.27)\end{array}$ & 0.1544 & $\begin{array}{l}0.67 \\
(0.29 \\
1.53)\end{array}$ & 0.3429 & $\begin{array}{l}2.67 \\
(0.75 \\
9.56)\end{array}$ & 0.1312 & $\begin{array}{l}1.67 \\
(0.57, \\
4.76)\end{array}$ & 0.3489 & $\begin{array}{l}4.76 \\
(0.74 \\
33.33)\end{array}$ & 0.1016 \\
\hline $\begin{array}{l}18.5 \sim \\
24 \mathrm{~kg} / \mathrm{m}^{2}\end{array}$ & $\begin{array}{l}0.95 \\
(0.84, \\
1.07)\end{array}$ & 0.3919 & $\begin{array}{l}1.21 \\
(0.73 \\
1.99)\end{array}$ & 0.4608 & $\begin{array}{l}1.25 \\
(1.12, \\
1.39)\end{array}$ & 0.0001 & $\begin{array}{l}1.14 \\
(0.88, \\
1.47)\end{array}$ & 0.3357 & $\begin{array}{l}0.91 \\
(0.68, \\
1.22)\end{array}$ & 0.5134 & $\begin{array}{l}0.58 \\
(0.17 \\
2.00)\end{array}$ & 0.3891 \\
\hline$\geq 24 \mathrm{~kg} / \mathrm{m}^{2}$ & $\begin{array}{l}0.88 \\
(0.79 \\
0.98)\end{array}$ & 0.0222 & $\begin{array}{l}0.86 \\
(0.60 \\
1.23)\end{array}$ & 0.4197 & $\begin{array}{l}1.14 \\
(1.06, \\
1.22)\end{array}$ & 0.0003 & $\begin{array}{l}1.37 \\
(1.19 \\
1.57)\end{array}$ & 0.0000 & $\begin{array}{l}1.22 \\
(0.88, \\
1.69)\end{array}$ & 0.2201 & $\begin{array}{l}4.35 \\
(0.83 \\
25.00)\end{array}$ & 0.0811 \\
\hline
\end{tabular}

$\mathrm{P}_{\mathrm{BMI}}$ interaction (Age, gender, BMI category, smoking status, alcohol intake status, diabetes status, hypertension status, TC/TG/HDL-C/LDL-C, and TC/TG,

$$
P_{T C * B M I} \text { category }=0.3627 \quad P_{T G^{*} B M I} \text { category }=0.2939 \quad P_{H D L-C * B M I \text { category }}=0.1011
$$

\begin{tabular}{|c|c|c|c|c|c|c|c|c|c|c|c|c|}
\hline No & $\begin{array}{l}0.93 \\
(0.84, \\
1.02)\end{array}$ & 0.1163 & $\begin{array}{l}1.10 \\
(0.77 \\
1.57)\end{array}$ & 0.5952 & $\begin{array}{l}1.23 \\
(1.15, \\
1.33)\end{array}$ & 0.0000 & $\begin{array}{l}1.30 \\
(1.11, \\
1.53)^{\prime}\end{array}$ & 0.0015 & $\begin{array}{l}1.03 \\
(0.81 \\
1.32)\end{array}$ & 0.8094 & $\begin{array}{l}0.84 \\
(0.23 \\
3.03)\end{array}$ & 0.7859 \\
\hline Yes & $\begin{array}{l}0.90 \\
(0.76 \\
1.05)\end{array}$ & 0.1858 & $\begin{array}{l}0.63 \\
(0.23 \\
1.73)\end{array}$ & 0.3731 & $\begin{array}{l}1.11 \\
(1.01, \\
1.23)\end{array}$ & 0.0330 & $\begin{array}{l}1.45 \\
(1.11, \\
1.89)^{\prime}\end{array}$ & 0.0063 & $\begin{array}{l}1.19 \\
(0.73 \\
1.96)\end{array}$ & 0.4848 & $\begin{array}{l}33.33 \\
(3.70, \\
373.82)\end{array}$ & 0.0022 \\
\hline
\end{tabular}

Smoking status (Age, gender, BMl, alcohol intake status, diabetes status, hypertension status, and TC/TG/HDL-C/LDL-C)

Abbreviations: BMI, body mass index; Cl, confidence interval; eGFR, estimated glomerular filtration rate; HDL-C, high-density lipoprotein-cholesterol; LDL-C, lon ratio; TC, total cholesterol; TG, triglycerides.

All calculations were weighted based on Chinese population data and the study sampling scheme. Multinomial logistic models were used to test the impact $\mathrm{a}$ for mildly impaired eGFR $\left(60 \sim 90 \mathrm{ml} / \mathrm{min}\right.$ per $\left.1.73 \mathrm{~m}^{2}\right)$ and moderately/severely impaired eGFR $\left(<60 \mathrm{ml} / \mathrm{min}\right.$ per $\left.1.73 \mathrm{~m}^{2}\right)$ in subpopulations stratified by ger intake status, diabetes status, and hypertension status, separately. In the overall cohort, the interaction terms were further included respectively to examine th in the multinomial logistic models were denoted in the parentheses. $P$ values $<0.05$ are shown in bold. 


\begin{tabular}{|c|c|c|c|c|c|c|c|}
\hline \multirow[t]{4}{*}{ Subpopulation } & \multicolumn{2}{|c|}{$\mathrm{TC}$, per $1 \mathrm{mmol} / \mathrm{L}$ increase } & \multicolumn{2}{|c|}{ TG, per $1 \mathrm{mmol} / \mathrm{L}$ increase } & \multicolumn{2}{|c|}{ HDL-C, per $1 \mathrm{mmol} / \mathrm{L}$ decrease } & l \\
\hline & $\begin{array}{l}\text { Mildly impaired } \\
\text { eGFR }\end{array}$ & $\begin{array}{l}\text { Moderately/severely } \\
\text { impaired eGFR }\end{array}$ & $\begin{array}{l}\text { Mildly impaired } \\
\text { eGFR }\end{array}$ & $\begin{array}{l}\text { Moderately/severely } \\
\text { impaired eGFR }\end{array}$ & $\begin{array}{l}\text { Mildly impaired } \\
\text { eGFR }\end{array}$ & $\begin{array}{l}\text { Moderately/severely } \\
\text { impaired eGFR }\end{array}$ & $\begin{array}{l}1 \\
\epsilon\end{array}$ \\
\hline & $\begin{array}{l}\text { (eGFR } 60 \sim \\
90 \mathrm{ml} / \mathrm{min} \text { per } \\
1.73 \mathrm{~m}^{2} \text { ) }\end{array}$ & $\begin{array}{l}(\text { eGFR }<60 \mathrm{ml} / \mathrm{min} \\
\left.\text { per } 1.73 \mathrm{~m}^{2}\right)\end{array}$ & $\begin{array}{l}\text { (eGFR } 60 \sim \\
90 \mathrm{ml} / \mathrm{min} \text { per } \\
1.73 \mathrm{~m}^{2} \text { ) }\end{array}$ & $\begin{array}{l}(\text { eGFR }<60 \mathrm{ml} / \mathrm{min} \\
\left.\text { per } 1.73 \mathrm{~m}^{2}\right)\end{array}$ & $\begin{array}{l}\text { (eGFR } 60 \sim \\
90 \mathrm{ml} / \mathrm{min} \text { per } \\
1.73 \mathrm{~m}^{2} \text { ) }\end{array}$ & $\begin{array}{l}(\text { eGFR }<60 \mathrm{ml} / \mathrm{min} \\
\left.\text { per } 1.73 \mathrm{~m}^{2}\right)\end{array}$ & ( \\
\hline & $\begin{array}{l}\text { OR } \\
(95 \% \mathrm{Cl})\end{array}$ & $\begin{array}{l}\text { OR } \\
(95 \% \mathrm{Cl})\end{array}$ & $\begin{array}{l}\text { OR } \\
(95 \% \mathrm{Cl})\end{array}$ & $\begin{array}{l}\text { OR } \\
(95 \% \mathrm{Cl})\end{array}$ & $\begin{array}{l}\text { OR } \\
(95 \% \mathrm{Cl})\end{array}$ & $\begin{array}{l}\text { OR } \\
(95 \% \mathrm{Cl})\end{array}$ & ( \\
\hline
\end{tabular}

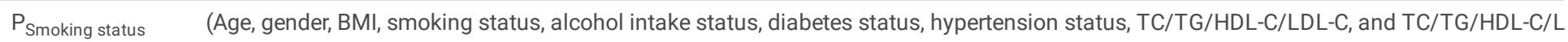
interaction
$P_{\text {TC*smoking status }}=0.6951$
$P_{T G^{\star} \text { smoking status }}=0.1753$
$P_{\text {HDL-C*smoking status }}=0.0219$

$r$

Alcohol intake (Age, gender, BMI, smoking status, diabetes status, hypertension status, and TC/TG/HDL-C/LDL-C) status

\begin{tabular}{|c|c|c|c|c|c|c|c|c|c|c|c|c|}
\hline No & $\begin{array}{l}0.91 \\
(0.83, \\
1.00)\end{array}$ & 0.0545 & $\begin{array}{l}1.10 \\
(0.77, \\
1.59)\end{array}$ & 0.5963 & $\begin{array}{l}1.22 \\
(1.14, \\
1.31)\end{array}$ & 0.0000 & $\begin{array}{l}1.32 \\
(1.14, \\
1.54)\end{array}$ & 0.0003 & $\begin{array}{l}0.92 \\
(0.72, \\
1.18)\end{array}$ & 0.4931 & $\begin{array}{l}0.80 \\
(0.22, \\
2.86)\end{array}$ & 0.7318 \\
\hline Yes & $\begin{array}{l}0.94 \\
(0.79 \\
1.12)\end{array}$ & 0.4837 & $\begin{array}{l}0.77 \\
(0.35, \\
1.68)\end{array}$ & 0.5135 & $\begin{array}{l}1.12 \\
(1.00 \\
1.24)\end{array}$ & 0.0444 & $\begin{array}{l}1.38 \\
(1.04, \\
1.83)\end{array}$ & 0.0274 & $\begin{array}{l}1.72 \\
(1.05 \\
2.86)\end{array}$ & 0.0296 & $\begin{array}{l}50.00 \\
(4.00 \\
557.18)\end{array}$ & 0.0023 \\
\hline
\end{tabular}

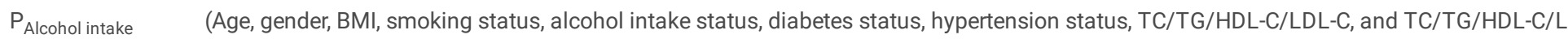
interaction
$P_{\text {TC*alcohol intake }}=0.6415$

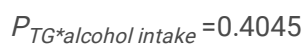

$P_{\text {HDL-C*alcohol intake }}=0.0007$

$t$

Diabetes status (Age, gender, BMI, smoking status, alcohol intake status, hypertension status, and TC/TG/HDL-C/LDL-C)

\begin{tabular}{|c|c|c|c|c|c|c|c|c|c|c|c|c|}
\hline $\begin{array}{l}\text { Normal } \\
\text { glycemic } \\
\text { metabolism }\end{array}$ & $\begin{array}{l}0.84 \\
(0.76, \\
0.93)\end{array}$ & 0.0005 & $\begin{array}{l}1.05 \\
(0.72 \\
1.53)\end{array}$ & 0.7989 & $\begin{array}{l}1.19 \\
(1.10, \\
1.29)\end{array}$ & 0.0000 & $\begin{array}{l}1.52 \\
(1.35, \\
1.72)\end{array}$ & 0.0000 & $\begin{array}{l}1.04 \\
(0.82, \\
1.33)\end{array}$ & 0.7152 & $\begin{array}{l}1.67 \\
(0.57 \\
5.00)\end{array}$ & 0.3456 \\
\hline Prediabetes & $\begin{array}{l}1.17 \\
(0.96 \\
1.43)\end{array}$ & 0.1139 & $\begin{array}{l}0.63 \\
(0.39 \\
1.02)\end{array}$ & 0.0587 & $\begin{array}{l}1.15 \\
(1.03, \\
1.29)\end{array}$ & 0.0170 & $\begin{array}{l}0.64 \\
(0.36, \\
1.14)\end{array}$ & 0.1295 & $\begin{array}{l}1.20 \\
(0.70 \\
2.08)\end{array}$ & 0.4889 & $\begin{array}{l}3.13 \\
(0.79 \\
12.50)\end{array}$ & 0.1060 \\
\hline Diabetes & $\begin{array}{l}0.86 \\
(0.71 \\
1.04)\end{array}$ & 0.1125 & $\begin{array}{l}1.64 \\
(0.86 \\
3.13)\end{array}$ & 0.1318 & $\begin{array}{l}1.19 \\
(1.04 \\
1.35)\end{array}$ & 0.0090 & $\begin{array}{l}1.48 \\
(1.21 \\
1.80)\end{array}$ & 0.0001 & $\begin{array}{l}1.05 \\
(0.56 \\
1.96)\end{array}$ & 0.8730 & $\begin{array}{l}0.59 \\
(0.05 \\
6.67)\end{array}$ & 0.6724 \\
\hline
\end{tabular}

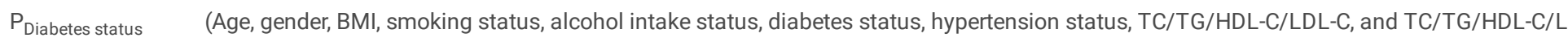
interaction
$P_{T C^{*} \text { diabetes status }}=0.0027$
$P_{\text {TG*diabetes status }}=0.0972$
$P_{H D L-C * \text { diabetes status }}=0.5666$

$t$

Hypertension (Age, gender, BMl, smoking status, alcohol intake status, diabetes status, and TC/TG/HDL-C/LDL-C)

\section{status}

\begin{tabular}{|c|c|c|c|c|c|c|c|c|c|c|c|c|}
\hline Normal tension & $\begin{array}{l}1.05 \\
(0.93 \\
1.19)\end{array}$ & 0.4355 & $\begin{array}{l}1.30 \\
(0.65 \\
2.58)\end{array}$ & 0.4553 & $\begin{array}{l}1.26 \\
(1.11, \\
1.43)\end{array}$ & 0.0003 & $\begin{array}{l}1.38 \\
(1.00, \\
1.90)\end{array}$ & 0.0480 & $\begin{array}{l}0.78 \\
(0.56 \\
1.09)\end{array}$ & 0.1467 & $\begin{array}{l}0.65 \\
(0.11 \\
3.85)\end{array}$ & 0.6314 \\
\hline Prehypertension & $\begin{array}{l}0.84 \\
(0.74 \\
0.95)\end{array}$ & 0.0059 & $\begin{array}{l}0.78 \\
(0.51, \\
1.19)\end{array}$ & 0.2477 & $\begin{array}{l}1.18 \\
(1.08, \\
1.28)\end{array}$ & 0.0001 & $\begin{array}{l}1.20 \\
(1.00 \\
1.43)\end{array}$ & 0.0481 & $\begin{array}{l}1.16 \\
(0.82 \\
1.67)\end{array}$ & 0.4006 & $\begin{array}{l}1.72 \\
(0.54 \\
5.56)\end{array}$ & 0.3531 \\
\hline Hypertension & $\begin{array}{l}0.86 \\
(0.72, \\
1.02)\end{array}$ & 0.0743 & $\begin{array}{l}1.06 \\
(0.64, \\
1.76)\end{array}$ & 0.8329 & $\begin{array}{l}1.12 \\
(1.01, \\
1.24)^{\prime}\end{array}$ & 0.0262 & $\begin{array}{l}1.33 \\
(1.08, \\
1.65)\end{array}$ & 0.0088 & $\begin{array}{l}1.35 \\
(0.85 \\
2.17)\end{array}$ & 0.1950 & $\begin{array}{l}1.33 \\
(0.15 \\
12.50)\end{array}$ & 0.7970 \\
\hline
\end{tabular}

$\mathrm{P}_{\text {Hypertension }} \quad$ (Age, gender, BMl, smoking status, alcohol intake status, diabetes status, hypertension status, TC/TG/HDL-C/LDL-C, and TC/TG/HDL-C/L status interaction
$P_{\text {TC*hypertension status }}=0.1081$
$P_{\text {TG*hypertension status }}=0.4163$

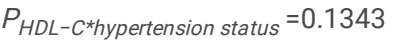

Abbreviations: BMI, body mass index; Cl, confidence interval; eGFR, estimated glomerular filtration rate; HDL-C, high-density lipoprotein-cholesterol; LDL-C, lon ratio; TC, total cholesterol; TG, triglycerides.

All calculations were weighted based on Chinese population data and the study sampling scheme. Multinomial logistic models were used to test the impact c for mildly impaired eGFR $\left(60 \sim 90 \mathrm{ml} / \mathrm{min}\right.$ per $\left.1.73 \mathrm{~m}^{2}\right)$ and moderately/severely impaired eGFR $\left(<60 \mathrm{ml} / \mathrm{min}\right.$ per $\left.1.73 \mathrm{~m}^{2}\right)$ in subpopulations stratified by ger intake status, diabetes status, and hypertension status, separately. In the overall cohort, the interaction terms were further included respectively to examine th in the multinomial logistic models were denoted in the parentheses. $P$ values $<0.05$ are shown in bold.

Significant interactions were observed between HDL-C and gender $\left(P_{H D L-C^{*} \text { gender }}=0.0003\right)$, smoking status $\left(P_{H D L-C^{*} \text { smoking status }}=0.0219\right)$, and alcohol intake

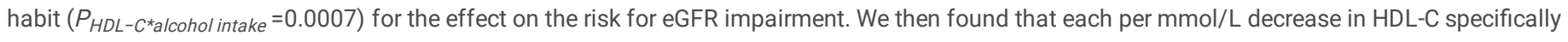


increased the risk for moderately/severely impaired eGFR by 20 - fold in males $(P=0.0001)$ and by 33.33-fold in smokers $(P=0.0022)$, but these associations were not identified in females $(P=0.2886)$ or those without a smoking habit $(P=0.7859)$. In individuals with an alcohol intake habit, each per mmol/L decrease in HDL-C was associated with 1.72-fold and 50.00-fold increases in the risks for mildly impaired and moderately/severely impaired eGFR, respectively $(P=0.0296,0.0023)$, and these associations were not observed in individuals without an alcohol intake habit $(P=0.4931,0.7318 ;$ Table 5$)$.

TC and diabetes status showed a significant interaction also $\left(P_{T C}{ }^{*}\right.$ diabetes status $\left.=0.0027\right)$. The association of elevated TC with a decreased risk for mildly impaired eGFR was only observed in individuals with normal glycemic metabolism [OR $(95 \% \mathrm{Cl}) 0.84(0.76,0.93), P=0.0005]$, and not in those with prediabetes $(P=0.1139)$ or diabetes $(P=0.1125)$. Moreover, TC did not show any association with the risk for moderately/severely eGFR impairment in individuals with normal glycemic metabolism $(P=0.7989)$, prediabetes $(P=0.0587)$, or diabetes $(P=0.1318$; Table 5$)$.

LDL-C and age showed significant interactions $\left(P_{L D L-C^{*} a g e}=0.0076\right)$. In those aged $40 \sim 60$ years, each per mmol/L increase in LDL-C was associated with decreased risks for mildly impaired eGFR $[0.84(0.75,0.94), P=0.0032]$ and moderately/severely impaired eGFR $[0.69(0.49,0.98), P=0.0382]$, but these associations were not observed in the participants aged $<40$ years $(P=0.1393,0.7755)$ or $\geq 60$ years $(P=0.2850,0.1833$; Table 5$)$.

\section{Discussion}

Epidemiological studies have suggested that dyslipidemia is associated with a reduced eGFR, but the specific types of lipids or lipoproteins that contribute to eGFR remained unclear for the general Chinese population. Based on the weighted data from a national representative population of 16,206 Chinese individuals, we found that the estimated mean eGFR values were significantly lower with TG levels in the abnormally high categories, and the prevalence rates of mildly impaired eGFR and moderately/severely impaired eGFR were significantly higher with TG levels in the abnormal categories (1.7 $2.3, \geq 2.3 \mathrm{mmol} / \mathrm{L}$ ). The estimated prevalence of mildly impaired eGFR was also higher with LDL-C levels in the borderline high category ( $\geq 4.1 \mathrm{mmol} / \mathrm{L})$. The results further indicate that high TG and low HDL-C were independently associated with a lower eGFR, whereas high TC and LDL-C were independently associated with a higher eGFR after adjustment for age, gender, BMI, smoking, alcohol intake, diabetes, and hypertension. Using multinomial logistic analyses, the TG level significantly contributed to the elevated risks for impaired eGFR. Moreover, a reduced HDL-C had a significantly stronger impact on eGFR in males, overweight/obese individuals, and those with smoking or alcohol intake habit. The findings of the present study highlight the importance of lipid and lipoprotein measurement in the risk assessment and prevention of impaired renal function in the general Chinese population, independent of the other conventional risk factors. A personalized management strategy for the at-risk population with recognition of the other conventional risk factors must be emphasized.

CKD is well known to be associated with an increased risk for cardiovascular events. In fact, previous studies indicated that a mild reduction in eGFR increases the risks for cardiovascular morbidity and mortality. For example, an observational study suggested that the risks for cardiovascular events and all-cause mortality increased in participants with an eGFR of $60 \sim 89 \mathrm{~mL} / \mathrm{min}$ per $1.73 \mathrm{~m}^{2}$ compared to those with an eGFR $\geq 90 \mathrm{~mL} / \mathrm{min}$ per $1.73 \mathrm{~m}{ }^{2}(20)$. In the Chinese population, the REACTION study reported that even a mildly reduced eGFR $\left(<90 \mathrm{~mL} / \mathrm{min}\right.$ per $\left.1.73 \mathrm{~m}^{2}\right)$ is associated with an elevated 10 -year Framingham risk for coronary heart disease and an elevated 10-year atherosclerotic cardiovascular disease risk among Chinese adults(2). At the same time, several studies established that monitoring of eGFR at a normal or milder stage is of clinical importance. In a community-based Chinese population aged over 40 years with normal or mildly impaired renal function, $12.3 \%$ of the participants developed renal function decline during a 4-year follow-up with a median change in the eGFR of $\sim 20 \mathrm{~mL} / \mathrm{min}$ per $1.73 \mathrm{~m}^{2}(21)$. Sumida et al. reported that $12 \%$ of the participants in a nationwide cohort of 56,946 United States veterans with an eGFR $\geq 60 \mathrm{~mL} / \mathrm{min}$ per $1.73 \mathrm{~m}^{2}$ showed rapid eGFR decline ( $\geq 5 \mathrm{ml} / \mathrm{min}$ per $1.73 \mathrm{~m}^{2} /$ year)(22). Interestingly, another study conducted in a US population found that 348 of 2,219 individuals with an eGFR of $60 \sim 119 \mathrm{~mL} / \mathrm{min}$ per $1.73 \mathrm{~m}^{2}$ showed rapid renal function decline ( $\geq 5 \mathrm{ml} / \mathrm{min}$ per $1.73 \mathrm{~m}^{2} /$ year) in an 8-year follow-up, and a greater percentage experienced rapid renal function decline among individuals with a baseline eGFR of $60 \sim$ $89 \mathrm{~mL} / \mathrm{min}$ per $1.73 \mathrm{~m}^{2}$ versus a baseline eGFR of $90 \sim 119 \mathrm{~mL} / \mathrm{min}$ per $1.73 \mathrm{~m}^{2}(23)$. These facts also support that individuals with mildly impaired renal function will experience more rapid renal function decline, making them major candidates for CKD in the future. In the present study, the estimated prevalence rates of both mildly impaired eGFR $\left(60 \sim 90 \mathrm{~mL} / \mathrm{min}\right.$ per $\left.1.73 \mathrm{~m}^{2}\right)$ and moderately/severely impaired eGFR $\left(<60 \mathrm{~mL} / \mathrm{min} \mathrm{per} 1.73 \mathrm{~m}{ }^{2}\right)$ were reported, and both outcomes were considered in the analyses applying the multinomial logistic procedure in SUDAAN software. Thus, the findings of the current study provide valuable information for the prevention of CKD in the general Chinese population aged 20 years and older.

Lipid disorder is proposed to be an essential cause of renal dysfunction, and patients with renal dysfunction usually have concomitant dyslipidemia. However, the link between lipid metabolism and the GFR have not been fully elucidated. Experimental data show that dyslipidemia can affect renal function and accelerate the progression of renal injury(24). Altered fatty acid and cholesterol metabolism are known as the key mediators of renal lipid accumulation, resulting in renal inflammation, oxidative stress, and fibrosis(25). Clinical studies showed that lipids or lipoproteins have discordant contributions to the GFR. Previous studies frequently showed that an increased TG level and a decreased HDL-C level were associated with a decreased eGFR, as well as the risk for renal dysfunction, in several different populations $(6,7,9-11)$. However, there has been a lack of research based on nationally representative general populations. In the current study, we estimated the prevalence rates and investigated the contributions of lipids and lipoproteins to eGFR and the risks for eGFR impairment in a nationally representative Chinese population with a large sample size, which was also weighted to represent the total population of Chinese adults on the basis of population data and sampling scheme. Thus, the results from this study can be reliably generalized to the general population of Chinese ancestry.

Our results demonstrated significant contributions of abnormal TG and HDL-C levels to decreased eGFR, as well as the contribution of high TG to elevated risks for both mildly impaired eGFR and moderately/severely impaired eGFR. The present study also emphasizes the importance of reducing TG and raising HDL-C for renal function protection in the general population. In this respect, drugs targeting TG or HDL-C, such as fibrate compounds, niacin, and inhibitors of cholesteryl ester transfer protein, might be beneficial theoretically. Evidence of their impacts on kidney function is still lacking. In current clinical practice, 
fibrate and niacin are only recommended for the treatment of patients with $\mathrm{TG} \geq 5.7 \mathrm{mmol} / \mathrm{L}$ to prevent pancreatitis, and for the treatment patients who were previously treated the maximum tolerable doses of statins and continued to have $T G \geq 2.6 \mathrm{mmol} / \mathrm{L}(18,26)$. However, in the FIELD Helsinki study, even concomitant decreases in creatinine clearance and eGFR in type 2 diabetes patients were revealed after long-term fenofibrate treatment for 5 years(27). Similar findings were also reported in outpatients not restricted to those with diabetes(28). In the future, clinical trials focusing on renal outcomes of drugs targeting TG or HDL-C are highly anticipated.

We previously reported that BMI, waist circumference, blood pressure, and fasting and post-prandial glucose levels are associated with TG in the Chinese population, whereas BMI, waist circumference, and glucose levels are associated with the HDL-C level(29). Interventions treating metabolic disorders are also well-known to be beneficial for the protection of renal function in Chinese populations. However, the findings of the present study indicate that TG and HDL-C influence renal function independent of the conventional risk factors including age, smoking and drink habits, as well as metabolic-related disorders, such as obesity, abnormal glycemic metabolism and hypertension. Thus, the present study highlights the critical impacts of TG and HDL-C on renal function beyond those conventional risk factors, indicating that these levels should be closely monitored and controlled.

Elevated TC and LDL-C levels are the predominant features of lipid disorder in patients with nephrotic syndrome. However, the exact influences of TC and LDL$\mathrm{C}$ on the eGFR continue to be debated. The conflicting results from different studies could have resulted from the differential adjustment for confounding factors or differences in the study populations. For instance, without controlling for the other conventional factors, negative correlations were observed between TC or LDL-C and eGFR in a Chinese population aged 40 years or older(7). In another study based on middle-aged and elder Chinese population, TC was associated with a decreased eGFR before and after adjustment for the conventional risk factors, and LDL-C was only associated with a decreased eGFR in the crude model(8). Moreover, other studies failed to identify any associations of TC or LDL-C with a decreased eGFR(9, 10). Notably, the CRIC study reported that a 1-standard deviation (SD) higher TC or LDL-C was associated with a $26 \%$ or $23 \%$ lower risk, respectively, of the renal end point after adjustment the other risk factors in patients with low levels of proteinuria (12). In the current study, among those with an abnormally high TC or LDL-C, the prevalence of eGFR impairment tended to be increased, suggesting that eGFR should be carefully monitored in individuals with abnormally high TC or LDL-C in clinical practice. On the other hand, after adjustment for the conventional risk factors, both elevated TC and LDL-C levels were independently associated with an increased eGFR, as well as lower risks for eGFR impairment. Although it cannot be determined whether causal relationships exist between TC or LDL-C and eGFR in the Chinese population via this cross-sectional study, it can be speculated that metabolic factors, such as BMI, diabetes status, and hypertension, have strong interplay with TC or LDL-C, leading to conflicting findings with or without adjustment for these factors. Moreover, the absolute roles of elevated TC and LDL-C independent of the other comorbid metabolic disorders may be protective of eGFR. In addition, statins, which are widely used to lower cholesterol levels by inhibiting the enzyme HMG-CoA reductase, have long been speculated to protect kidney function $(4,5)$. A recent meta-analysis conducted in 2016 reported that statin therapy does not reduce the risk for kidney failure events in adults not receiving dialysis, but may modestly reduce proteinuria and the rate of eGFR decline(30). Thus, the exact biological effects of TC and LDL-C on the eGFR remain incompletely understood.

Using interaction and subpopulation analyses, a decreased HDL-C was observed to have significantly stronger influences on the reduction of eGFR and elevated risks for impaired eGFR in males and individuals with smoking or alcohol intake habit, as well as a stronger influence on the reduction of eGFR in overweight/obese individuals. These results suggest that the disturbance of HDL-C can be more harmful, especially for these patient groups. In the Chinese population, the majority of the individuals with smoking or alcohol intake habits are men, which may partly explain the similar patterns of the interacting effects of these factors. Notably, because smoking and alcohol intake are modifiable factors, lifestyle intervention, such as smoking cession and alcohol avoidance, might reduce an individual's risk for renal dysfunction through the interaction with HDL-C. Based on the observation that the eGFR of overweight or obese individuals was more susceptible to the reduction of $\mathrm{HDL}-\mathrm{C}$, these patients may also benefit from weight loss intervention. Moreover, each per mmol/L TG increase significantly increased the risk for moderately/severely impaired eGFR by $45 \%$ specifically in males, but not in females, suggesting that males were more susceptible to the effects of increased TG. We also found that the protective role of TC against mildly impaired eGFR did not exist among individuals with prediabetes or diabetes. Also, the protective effect of LDL-C was observed only in the middle-aged population (40 60 years old). Overall, the interaction data highlight the necessity of personalized management of the eGFR for the prevention of CKD.

The current study has the following strengths. The present study was based on a relatively large nationally representative survey in China, and the data were weighted on basis of the population data and sampling strategy, so that the results could be well-generalized to the Chinese population. Second, the mild eGFR impairment was taken into consideration by applying a multinomial logistic regression analysis, which provided valuable data for the early prevention and intervention of renal function decline. Finally, the elucidation of interaction effects of conventional factors can be applied to achieve precise risk assessment and improve intervention strategies. However, this study also has limitations. As a cross-sectional survey, it could not elucidate the sequential effects of the onset of dyslipidemia and eGFR impairment. Therefore, longitudinal studies are required to test the robustness of the findings of this study. Moreover, accumulating data for the involvement of lipid composites besides TC, TG, HDL-C, and LDL-C are found in the literatures(31, 32), suggesting that novel lipid biomarkers for eGFR not covered in the present study still need to be investigated.

In summary, we investigated the contributions of the four clinical measurements of lipids and lipoproteins on eGFR, as well as the risks for mild eGFR reduction and moderate/severe eGFR reduction based on weighted data from a nationally representative general population of Chinese ancestry. Significant increases in TG, TC, and LDL-C were accompanied by a decrease in the eGFR. With adjustment for the conventional risk factors, elevated TG and reduced HDL$C$ were independently associated with impaired eGFR outcomes, whereas TC and LDL-C levels were positively associated with the eGFR. Moreover, the effects of a lower HDL-C on eGFR reduction were significantly stronger in males, those with smoking or alcohol intake habits, and overweight/obese individuals, while males were more susceptible to the effect of increased TG on the risk for a moderately/severely impaired eGFR. These findings emphasize the importance of effective control of TG and HDL-C levels for protecting renal function in Chinese adults aged 20 years and above, and the need for the development of a personalized management strategy for the at-risk population. In this respect, individuals with abnormal TG or HDL-C levels require close monitoring of the eGFR and should receive effective interventions to reduce TG and increase HDL-C. 


\section{List Of Abbreviations}

2-h PPG, 2-h post-prandial plasma glucose; $\beta$, beta coefficient; $\mathrm{Cl}$, confidence interval; CKD, chronic kidney disease; DBP, diastolic blood pressure; DMS, the China National Diabetes and Metabolism Disorders Study; eGFR, estimated glomerular filtration rate; ESRD, end-stage renal disease; FPG, fasting plasma glucose; HDL-C, high-density lipoprotein-cholesterol; LDL-C, low-density lipoprotein-cholesterol; MDRD, Modification of Diet in Renal Disease; OGTT, oral glucose tolerance test; OR, odds ratio; SBP, systolic blood pressure; SE, standard error; TC, total cholesterol; TG, triglycerides; UACR, urinary albumin-creatinine ratio; WHO, World Health Organization.

\section{Declarations}

Ethics approval and consent to participate

The study protocol was conducted in accordance with the Declaration of Helsinki II and approved by the ethics committee of the China-Japan Friendship Hospital (Beijing, China). Written informed consent was obtained from each participant prior to data collection.

\section{Availability of data and materials}

All data generated or analyzed during this study are included in this published article.

\section{Competing interests}

We also have following competing interest to disclose. Outside the submitted work, WY has attended the advisory board of Novo Nordisk, received investigator-initiated trial research funds from AstraZeneca, and been a speaker for Novo Nordisk, Bayer, Sanofi Aventis, Merck Sharp \& Dohme China, AstraZeneca, Eli Lilly, Boehringer-Ingelheim, and Servier; received honorarium and travel support as the advisory boards' member from Merck \& Co., Inc. These do not alter our adherence to all the journal policies on sharing data and materials, as detailed online in the guide for authors.

\section{Funding}

This work was supported by grants from the Ministry of Science and Technology of the People's Republic of China (2016YFC0901204), the Chinese Medical Association Foundation, and Sanofi (China) Investment Company Limited. There are no patents, products in development or marketed products to declare. The funders had no role in study design, data collection and analysis, decision to publish, or preparation of the manuscript.

\section{Authors' contributions}

All authors contributed significantly and met the criteria of authorship. XC and XK generated the study hypothesis, and developed the study design. BZ, XC, XX, $\mathrm{HZ}$, and $\mathrm{XK}$ collected the data. $\mathrm{XK}, \mathrm{XC}, \mathrm{ZY}, \mathrm{LY}$, and $\mathrm{WY}$ analyzed and interpreted the data. XK drafted the manuscript. All of the authors critically revised the manuscript and contributed to the discussion. XC completed the final version of manuscript. All authors are in agreement with the content of the manuscript, and agree to submit for consideration for publication in the journal. There are no any ethical/legal conflicts involved in the article. As the corresponding author, $\mathrm{XC}$ have had full access to the data in the study and final responsibility for the decision to submit for publication.

\section{Acknowledgments}

We thank all the patients for their participation in the study. We thank all the staff of the DMS who contributed to recruiting participants and obtaining samples.

This work was supported by grants from the Ministry of Science and Technology of the People's Republic of China (2016YFC0901204) (X.C.) and the Chinese Medical Association Foundation (http://en.cma.org.cn) (W.Y.). This study was also partly funded by Sanofi (China) Investment Company Limited (http://www.sanofi.cn/l/cn/zh/index.jsp) (W.Y.).

\section{References}

1. Zhang L, Wang F, Wang L, Wang W, Liu B, Liu J, Chen M, He Q, Liao Y, Yu X, Chen N, Zhang JE, Hu Z, Liu F, Hong D, Ma L, Liu H, Zhou X, Chen J, Pan L, Chen W, Wang W, Li X, Wang H. Prevalence of chronic kidney disease in China: a cross-sectional survey. Lancet. 2012;379(9818):815-22.

2. Lu J, Mu Y, Su Q, Shi L, Liu C, Zhao J, Chen L, Li Q, Yang T, Yan L, Wan Q, Wu S, Liu Y, Wang G, Luo Z, Tang X, Chen G, Huo Y, Gao Z, Ye Z, Wang Y, Qin G, Deng H, Yu X, Shen F, Chen L, Zhao L, Sun J, Sun W, Wang T, Du R, Lin L, Dai M, Xu Y, Xu M, Bi Y, Lai S, Li D, Wang W, Ning G. Reduced Kidney Function Is Associated With Cardiometabolic Risk Factors, Prevalent and Predicted Risk of Cardiovascular Disease in Chinese Adults: Results From the REACTION Study. J. Am. Heart Assoc. 2016; 5(7).

3. Natali A, Boldrini B, Baldi S, Rossi M, Landi P, Severi S, Solini A, Ferrannini E. Impact of mild to moderate reductions of glomerular filtration rate on coronary artery disease severity. Nutr Metab Cardiovasc Dis. 2014;24(6):681-8.

4. Cholesterol Treatment Trialists' Collaboration. Efficacy and safety of statin therapy in older people: a meta-analysis of individual participant data from 28 randomised controlled trials. Lancet. 2019;393(10170):407-15.

5. Herrington WG, Emberson J, Mihaylova B, Blackwell L, Reith C, Solbu MD, Mark PB, Fellstrom B, Jardine AG, Wanner C, Holdaas H, Fulcher J, Haynes R, Landray MJ, Keech A, Simes J, Collins R, Baigent C. Impact of renal function on the effects of LDL cholesterol lowering with statin-based regimens: a meta-analysis of individual participant data from 28 randomised trials. Lancet Diabetes Endocrinol. 2016;4(10):829-39. 
6. Zheng W, Qian G, Hao W, Geng X, Hong Q, Cai G, Chen X. Wu, D. Cardiovascular metabolic risk factors and glomerular filtration rate: a rural Chinese population study. Lipids Health Dis. 2016;15(1):180.

7. Sun K, Lin D, Li F, Huang C, Qi Y, Xue S, Tang J, Yang C, Li Y, Ren M. Yan, L. Discordant associations of lipid parameters with albuminuria and chronic kidney disease: a population-based study. Lipids Health Dis. 2015; 14(1).

8. Hou X, Wang C, Zhang X, Zhao X, Wang Y, Li C, Li M, Wang S, Yang W, Ma Z, Ma A, Zheng H, Wu J, Sun Y, Song J, Lin P, Liang K, Gong L, Wang M, Liu F, Li W, Xiao J, Yan F, Yang J, Wang L, Tian M, Liu J, Zhao R, Chen S. Chen, L. Triglyceride levels are closely associated with mild declines in estimated glomerular filtration rates in middle-aged and elderly Chinese with normal serum lipid levels. Plos One. 2014;9(9):e106778.

9. Muntner P, Coresh J, Smith JC, Eckfeldt J, Klag MJ. Plasma lipids and risk of developing renal dysfunction: the atherosclerosis risk in communities study. Kidney Int. 2000;58(1):293-301.

10. Tozawa M, Iseki K, Iseki C, Oshiro S, Ikemiya Y, Takishita S. Triglyceride, but not total cholesterol or low-density lipoprotein cholesterol levels, predict development of proteinuria. Kidney Int. 2002;62(5):1743-9.

11. Samuelsson O, Attman PO, Knight-Gibson C, Larsson R, Mulec H, Weiss L, Alaupovic P. Complex apolipoprotein B-containing lipoprotein particles are associated with a higher rate of progression of human chronic renal insufficiency. J Am Soc Nephrol. 1998;9(8):1482-8.

12. Rahman M, Yang W, Akkina S, Alper A, Anderson AH, Appel LJ, He J, Raj DS, Schelling J, Strauss L, Teal V, Rader DJ. Relation of serum lipids and lipoproteins with progression of CKD: The CRIC study. Clin J Am Soc Nephrol. 2014;9(7):1190-8.

13. Yang W, Lu J, Weng J, Jia W, Ji L, Xiao J, Shan Z, Liu J, Tian H, Ji Q, Zhu D, Ge J, Lin L, Chen L, Guo X, Zhao Z, Li Q, Zhou Z, Shan G, He J. Prevalence of diabetes among men and women in China. N Engl J Med. 2010;362(12):1090-101.

14. Ma YC, Zuo L, Chen JH, Luo Q, Yu XQ, Li Y, Xu JS, Huang SM, Wang LN, Huang W, Wang M, Xu GB, Wang HY. Modified glomerular filtration rate estimating equation for Chinese patients with chronic kidney disease. J Am Soc Nephrol. 2006;17(10):2937-44.

15. Levey AS, Coresh J, Balk E, Kausz AT, Levin A, Steffes MW, Hogg RJ, Perrone RD, Lau J, Eknoyan G. National Kidney Foundation practice guidelines for chronic kidney disease: evaluation, classification, and stratification. Ann Intern Med. 2003;139(2):137-47.

16. Wang YX, Wang AP, Ye YN, Gao ZN, Tang XL, Yan L, Wan Q, Wang WQ, Luo ZJ, Qin GJ, Chen LL, Mu YM. Elevated triglycerides rather than other lipid parameters are associated with increased urinary albumin to creatinine ratio in the general population of China: a report from the REACTION study. Cardiovasc Diabetol. 2019;18(1):57.

17. Expert Panel on Detection. Evaluation, and Treatment of High Blood Cholesterol in Adults. Executive Summary of The Third Report of The National Cholesterol Education Program (NCEP) Expert Panel on Detection, Evaluation, And Treatment of High Blood Cholesterol In Adults (Adult Treatment Panel III). JAMA. 2001;285(19):2486-97.

18. Joint committee issued Chinese guideline for the management of dyslipidemia in. adults. 2016 Chinese guideline for the management of dyslipidemia in adults. Zhonghua Xin Xue Guan Bing Za Zhi. 2016;44(10):833-53.

19. Chobanian AV, Bakris GL, Black HR, Cushman WC, Green LA, Izzo JJ, Jones DW, Materson BJ, Oparil S, Wright JJ, Roccella EJ. The Seventh Report of the Joint National Committee on Prevention, Detection, Evaluation, and Treatment of High Blood Pressure: the JNC 7 report. JAMA. 2003;289(19):2560-72.

20. Mohandas R, Segal M, Srinivas TR, Johnson BD, Wen X, Handberg EM, Petersen JW, Sopko G, Merz CN, Pepine CJ. Mild renal dysfunction and long-term adverse outcomes in women with chest pain: results from the National Heart, Lung, and Blood Institute-sponsored Women's Ischemia Syndrome Evaluation (WISE). Am Heart J. 2015;169(3):412-8.

21. Wang F, Zhang L, Zuo L, Liu L, Wang H. Mortality and renal function decline among a community-based Chinese population with normal or mildly impaired renal function. Nephrol Dial Transpl. 2011;26(9):2847-52.

22. Sumida K, Molnar MZ, Potukuchi PK, George K, Thomas F, Lu JL, Yamagata K, Kalantar-Zadeh K, Kovesdy CP. Changes in Albuminuria and Subsequent Risk of Incident Kidney Disease. Clin J Am Soc Nephrol. 2017;12(12):1941-9.

23. Koraishy FM, Hooks-Anderson D, Salas J, Rauchman M, Scherrer JF. Fast GFR decline and progression to CKD among primary care patients with preserved GFR. Int Urol Nephrol. 2018;50(3):501-8.

24. Izquierdo-Lahuerta A, Martinez-Garcia C, Medina-Gomez G. Lipotoxicity as a trigger factor of renal disease. J Nephrol. 2016;29(5):603-10.

25. D'Agati VD, Chagnac A, de Vries AP, Levi M, Porrini E, Herman-Edelstein M, Praga M. Obesity-related glomerulopathy: clinical and pathologic characteristics and pathogenesis. Nat Rev Nephrol. 2016;12(8):453-71.

26. CLINICAL ENDOCRINOLOGISTS AND AMERICAN COLLEGE OF ENDOCRINOLOGY GUIDELINES FOR MANAGEMENT OF DYSLIPIDEMIA AND PREVENTION OF CARDIOVASCULAR DISEASE

Jellinger PS, Handelsman Y, Rosenblit PD, Bloomgarden ZT, Fonseca VA, Garber AJ, Grunberger G, Guerin CK, Bell D, Mechanick JI, Pessah-Pollack R, Wyne $\mathrm{K}$, Smith D, Brinton EA, Fazio S, Davidson M, Zangeneh F, Bush MA, AMERICAN ASSOCIATION OF. CLINICAL ENDOCRINOLOGISTS AND AMERICAN COLLEGE OF ENDOCRINOLOGY GUIDELINES FOR MANAGEMENT OF DYSLIPIDEMIA AND PREVENTION OF CARDIOVASCULAR DISEASE - EXECUTIVE SUMMARY Complete Appendix to Guidelines available at http://journals.aace.com. Endocr. Pract. 2017; 23(4): 479-497.

27. Forsblom C, Hiukka A, Leinonen ES, Sundvall J, Groop PH, Taskinen MR. Effects of long-term fenofibrate treatment on markers of renal function in type 2 diabetes: the FIELD Helsinki substudy. Diabetes Care. 2010;33(2):215-20.

28. Kim S, Ko K, Park S, Lee DR, Lee J. Effect of Fenofibrate Medication on Renal Function. Korean J Fam Med. 2017;38(4):192-8.

29. Yang W, Xiao J, Yang Z, Ji L, Jia W, Weng J, Lu J, Shan Z, Liu J, Tian H, Ji Q, Zhu D, Ge J, Lin L, Chen L, Guo X, Zhao Z, Li Q, Zhou Z, Shan G, He J. Serum lipids and lipoproteins in Chinese men and women. Circulation. 2012;125(18):2212-21.

30. Su X, Zhang L, Lv J, Wang J, Hou W, Xie X, Zhang H. Effect of Statins on Kidney Disease Outcomes: A Systematic Review and Meta-analysis. Am J Kidney Dis. 2016;67(6):881-92. 
31. Kim OY, Lee SM, An WS. Impact of Blood or Erythrocyte Membrane Fatty Acids for Disease Risk Prediction: Focusing on Cardiovascular Disease and Chronic Kidney Disease. Nutrients. 2018; 10(10).

32. Hocher B, Adamski J. Metabolomics for clinical use and research in chronic kidney disease. Nat Rev Nephrol. 2017;13(5):269-84. 